\title{
REGIONS OF MULTISTATIONARITY IN CASCADES OF GOLDBETER-KOSHLAND LOOPS
}

\author{
MAGALÍ GIAROLI, FRÉDÉRIC BIHAN, AND ALICIA DICKENSTEIN
}

\begin{abstract}
We consider cascades of enzymatic Goldbeter-Koshland loops [19] with any number $n$ of layers, for which there exist two layers involving the same phosphatase. Even if the number of variables and the number of conservation laws grow linearly with $n$, we find explicit regions in reaction rate constant and total conservation constant space for which the associated mass-action kinetics dynamical system is multistationary. Our computations are based on the theoretical results of our companion paper [4], which are inspired by results in real algebraic geometry by Bihan, Santos and Spaenlehauer [5].
\end{abstract}

\section{INTRODUCTION}

Signal transduction is the process through which cells communicate with the external environment, interpret stimuli and respond to them. This mechanism is controlled by signaling cascades. Classical signaling pathways typically contain a cascade of phosphorylation cycles where the activated protein in one layer acts as the modifier enzyme in the next layer. An example of signaling cascades is the Ras cascade (see Figure 1, as it is usually depicted in the biochemistry literature), which is an important signaling pathway in mitogen-activated protein kinases (MAPKs). This cascade reaction activates transcription factors and regulates gene expression. The Ras signaling pathway has a significant role in the occurrence and development of diseases such as cancer [24] or developmental defects [20]. One key property is the occurrence of multistability, which triggers different crucial cellular events. A basic condition for these different cellular responses is the emergence of multistationarity.

A reaction network $G$ on a given set of $s$ chemical species is a finite directed graph whose edges $\mathcal{R}$ represent the reactions and are labeled by parameters $\kappa \in \mathbb{R}_{>0}^{|\mathcal{R}|}$, known as reaction rate constants, and whose vertices are labeled by complexes, usually represented as nonnegative integer linear combinations of species. After numbering the species, a complex can be identified with a vector in $\mathbb{Z}_{>0}^{s}$. Under mass-action kinetics, $G$ defines the following autonomous system of ordinary differential equations in the concentrations $x_{1}, x_{2}, \ldots, x_{s}$ of the species as functions of time $t$ :

$$
\dot{x}=f(x)=\left(\frac{d x_{1}}{d t}, \frac{d x_{2}}{d t}, \ldots, \frac{d x_{s}}{d t}\right)=\sum_{y \rightarrow y^{\prime} \in \mathcal{R}} \kappa_{y y^{\prime}} x^{y}\left(y^{\prime}-y\right),
$$

where $x=\left(x_{1}, x_{2}, \ldots, x_{s}\right), f=\left(f_{1}, \ldots, f_{s}\right), x^{y}=x_{1}^{y_{1}} x_{2}^{y_{2}} \ldots x_{s}^{y_{s}}$ and $y \rightarrow y^{\prime}$ indicates that the complex $y$ reacts to the complex $y^{\prime}$ and $\left(y, y^{\prime}\right) \in \mathcal{R}$. The steady states of the system correspond to constant trajectories, that is, to the common zero set of the polynomials $f_{1}, \ldots, f_{s} \in \mathbb{R}\left[x_{1}, \ldots, x_{s}\right]$. As the vector $\dot{x}(t)$ lies for all time $t$ in the linear subspace $S$ spanned by the reaction vectors $\left\{y^{\prime}-y: y \rightarrow y^{\prime} \in \mathcal{R}\right\}$ (which is known as the stoichiometric subspace), it follows that any trajectory $x(t)$ lies in a translate of $S$. Moreover, if $x(0)=$ $x^{0} \in \mathbb{R}_{>0}^{s}$, then $x(t)$ lies for any $t$ (in the domain of definition) in the stoichiometric compatibility class $\left(x^{0}+S\right) \cap \mathbb{R}_{\geqslant 0}^{s}$. We will work with conservative systems and so all trajectories will be defined for any $t \geq 0$. The linear equations of $x^{0}+S$ give conservation

\footnotetext{
Key words and phrases. Enzymatic cascades, Goldbeter-Koshland loops, sparse polynomial systems, multistationarity.

AD and MG are partially supported by UBACYT 20020100100242, CONICET PIP 11220150100473, and ANPCyT PICT 2013-1110, Argentina.
} 


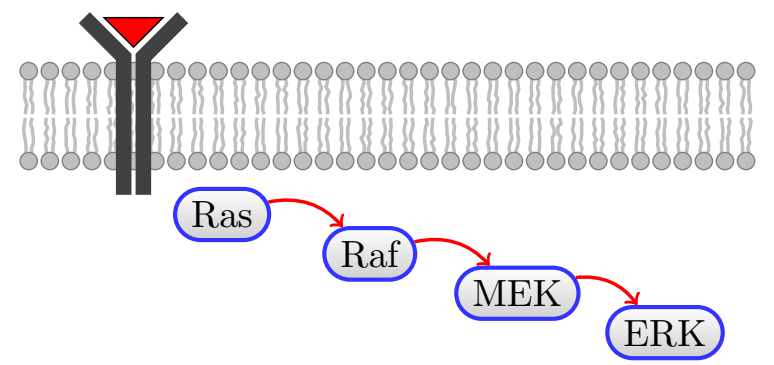

Figure 1. The Ras pathway.

laws. If $x^{0} \in \mathbb{R}_{>0}^{s}$, we can also write the linear variety $x^{0}+S$ in the form: $\left\{x \in \mathbb{R}^{s}\right.$ : $\left.\ell_{1}(x)=T_{1}, \ldots, \ell_{\sigma}(x)=T_{\sigma}\right\}$, where $\ell_{1}, \ldots, \ell_{\sigma}$ are linear forms defining a basis of the subspace orthogonal to $S$ and $T=\left(T_{1}, \ldots, T_{\sigma}\right) \in \mathbb{R}_{\geq 0}^{\sigma}$. These constant values are called total conservation constants.

The network $G$ is said to have the capacity for multistationarity if there exists a choice of reaction rate constants $\kappa$ and total conservation constants $T$ such that there are two or more steady states of system (1.1) in the stoichiometric compatibility class determined by $T$. Several articles studied the capacity for multistationarity from the structure of the directed graph of reactions $[1,15,16,17,22,25,26]$, a line initiated in [10, 11]. When the capacity for multistationarity of $G$ is determined, the following difficult step is to find values of multistationary parameters. This is a question of quantifier elimination in real algebraic geometry, which is effective, but for interesting networks the complexity of the computations with general standard tools is too high. Several articles in the literature addressed this question, with different approaches based on ad-hoc computations, injectivity results which use signs of minors in different forms, degree theory $[7,8,9,21,23,28]$ and the study of sparse real polynomials [18].

In this work, we use tools from real algebraic geometry based on the papers $[4,5]$, to analyze multistationarity in cascades of enzymatic Goldbeter-Koshland loops. A second important ingredient of our approach is the observation that enzymatic cascades have the structure of MESSI systems, introduced and studied in [26], from which an explicit parametrization of the steady states can be obtained, even in presence of multistationarity. We show how to deform a given set of parameters of the model to produce multistationarity, including both the reaction rate constants and the total concentration constants. Moreover, we identify open sets where multistationarity occurs in the space of all these parameters.

In Section 2 we state and explain the theoretical setting presented in our companion paper [4], based on the results in [5]. We apply our method to enzymatic cascades of Goldbeter-Koshland loops in Sections 3 and 4. In Section 3 we apply our method to an enzymatic cascade with two layers and in Section 4 we work with the general case of $n$ layers and present our main results (Theorems 4.1 and 4.3.) In this case, the associated polynomial systems have positive dimensions growing linearly with $n$. The number of conservation relations (and then of total conservation constants) also grows linearly with $n$, and it is at least four if $n \geq 2$. Such systems were studied in $[6,14]$ when all the enzymes are different, in which case there cannot be more than one positive steady state. This fact is proved in [14] and also is a particular case of a more general result in [3], in which the authors work with a more general structure: tree networks of Goldbeter-Koshland loops. In the case of two layers, it was shown in [15] that in the case $n=2$ (see Figure 2), if the same phosphatase is acting at both layers, then the network has the capacity for multistationarity. It can be deduced from the results in [1], that if there are any number of layers, and the last two share a phosphatase, multistationarity parameters for the case $n=2$ can be extended to produce multistationarity parameters in the general case.

Our approach allows us to describe open sets in the rate constant and total concentration parameters which ensure multistationarity as long as at any pair of layers in the 
(A)

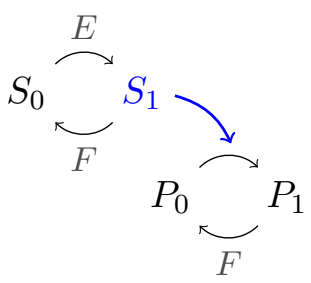

(B)

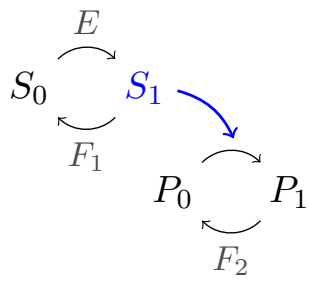

Figure 2. Same and different phosphatases in a 2-layer cascade of GK-loops.

cascade there is a shared phosphatase. Note for instance the structure of the statement of Theorem 4.1: if the given rate constants verify inequality (4.3), then we give explicit inequalities on the total conservation constants for which multistationarity occurs after tuning some of the reaction rate constants not involved in (4.3), that we clearly specify.

Our results can be generalized to describe multistationarity regions for other architectures of cascades which define MESSI systems. For this purpose, we state and prove in an Appendix the extension Theorem A.3, that abstracts some of our computations in Section 4. We also refer to the general results in Section 5 in [4]. For example, in the case of the Ras cascade in Figure 1, previous papers studied rate constant multistationarity parameters (see e.g. $[8,27]$ ). Our methods yield multistationarity regions for this signaling pathway in terms of rate constants and total conservation parameters. We omit these computations, which are similar to the ones we detail in Sections 3 and 4.

\section{Positive SOlutions of SPARSe POlynomial Systems}

We summarize some results from Section 2 in [4], where complete details can be found. We also refer the reader to [12] for the combinatorial objects that we introduce in this section.

We consider a polynomial system of $d$ Laurent polynomial equations $f_{1}(x)=\cdots=$ $f_{d}(x)=0$ in $d$ variables $x=\left(x_{1}, \ldots, x_{d}\right)$, with

$$
f_{i}(x)=\sum_{j=1}^{n} c_{i j} x^{a_{j}} \in \mathbb{R}\left[x_{1}, \ldots, x_{d}\right], i=1, \ldots, d,
$$

where the exponents belong to a fixed finite point configuration $\mathcal{A}=\left\{a_{1}, \ldots, a_{n}\right\} \subset \mathbb{Z}^{d}$, with $n+2 \geq d$. We denote by $C=\left(c_{i j}\right) \in \mathbb{R}^{d \times n}$ the coefficient matrix of the system and we assume w.l.o.g. that no column of $C$ is identically zero. The set $\mathcal{A}$ is called the support of the polynomial system.

In order to understand the notation we present a basic example.

Example 2.1. We consider the following point configuration

$$
\mathcal{A}=\{(0,0),(2,0),(0,1),(2,1),(1,2),(1,3)\} \subset \mathbb{Z}^{2},
$$

and the coefficient matrix

$$
C=\left(\begin{array}{rrrrrr}
1 & -2 & 1 & 1 & -1 & 0 \\
-2 & 1 & 0 & -1 & -1 & 1
\end{array}\right)
$$

The polynomial system of two polynomial equations and two variables $x, y$ :

$$
\begin{aligned}
1-2 x^{2}+y+x^{2} y-x y^{2} & =0, \\
-2+x^{2}-x^{2} y-x y^{2}+x y^{3} & =0,
\end{aligned}
$$

has support $\mathcal{A}$ and coefficient matrix $C$, and we write it in the form:

$$
C\left(1 \quad x^{2} \quad y \quad x^{2} y \quad x y^{2} \quad x y^{3}\right)^{t}=0,
$$

where $t$ denotes the transpose. 


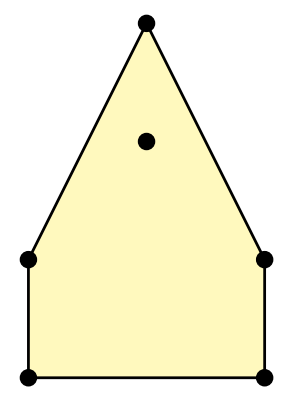

Figure 3. Convex hull of the support $\mathcal{A}$ of the Example 2.1.

Our idea to ensure multistationarity, based on [5], is to restrict our polynomial system (2.1) to subsystems which have a positive solution and then extend these solutions to the total system, under a deformation of the coefficients. So, we are first interested in finding conditions in the coefficient matrix that guarantee a positive solution in the subsystems.

Suppose that the convex hull of $\mathcal{A}$ is a full-dimensional polytope $Q$. Figure 3 shows the convex hull of $\mathcal{A}$ of Example 2.1. A $d$-simplex with vertices in $\mathcal{A}$ is a subset of $d+1$ points of $\mathcal{A}$ affinely independent. Following Section 3 in [5], we define:

Definition 2.2. Given any $d \times(d+1)$ matrix $M$ with real entries, we denote by $\operatorname{minor}(M, i)$ the determinant of the square matrix obtained by removing the $i$-th column. The matrix $M$ is called positively spanning if all the values $(-1)^{i} \operatorname{minor}(M, i)$, for $i=1, \ldots, d+1$, are nonzero and have the same sign.

Thus, a matrix is positively spanning if all the coordinates of any non-zero vector in the kernel of the matrix are non-zero and have the same sign. For example, the matrix

$$
M=\left(\begin{array}{lll}
1 & 0 & -1 \\
0 & 1 & -1
\end{array}\right)
$$

is positively spanning because the values $(-1)^{1} \operatorname{minor}(M, 1)=-1,(-1)^{2} \operatorname{minor}(M, 2)=$ -1 and $(-1)^{3} \operatorname{minor}(M, 3)=-1$ are all nonzero and have the same sign. Also, any nonzero vector in the kernel of $M$ is of the form $\lambda(1,1,1)$, with $\lambda \in \mathbb{R}-\{0\}$, that is, all the coordinates of one of these vectors share the same sign.

It can be shown (Proposition 3.3 in [5]) that if a polynomial system of $d$ polynomial equations in $d$ variables has a $d$-simplex as support, then it has one non-degenerate positive solution if and only if its $d \times(d+1)$ matrix of coefficients is positively spanning.

Definition 2.3. Let $C$ be $a d \times n$ matrix with real entries. $A d$-simplex $\Delta=\left\{a_{i_{1}}, \ldots, a_{i_{d+1}}\right\}$ is said to be positively decorated by $C$ if the $d \times(d+1)$ submatrix of $C$ with columns $\left\{i_{1}, \ldots, i_{d+1}\right\}$ is positively spanning.

Example 2.4. (Example 2.1 continued). Consider again the support with vertices in $\mathcal{A}$ in (2.2), and the following 2-simplices:

$$
\Delta_{1}=\{(0,0),(2,0),(0,1)\}, \quad \Delta_{2}=\{(2,0),(0,1),(2,1)\}, \quad \Delta_{3}=\{(0,1),(2,1),(1,2)\},
$$

depicted in Figure 6. The submatrix of $C$ given by the columns of $\Delta_{1}$ (the first three columns) equals:

$$
\left(\begin{array}{rrr}
1 & -2 & 1 \\
-2 & 1 & 0
\end{array}\right)
$$

This matrix is positively spanning, so the simplex $\Delta_{1}$ is positively decorated by $C$. It is easy to check that the simplex $\Delta_{2}$ is also positively decorated by $C$. But the submatrix given by the columns of $\Delta_{3}$ (the columns 3,4 and 5 ):

$$
\left(\begin{array}{rrr}
1 & 1 & -1 \\
0 & -1 & -1
\end{array}\right)
$$

is not positively spanning, and then the simplex $\Delta_{3}$ is not positively decorated by $C$. 
Given a $d$-simplex $\Delta$ with vertices in $\mathcal{A}$, we consider height vectors $h \in \mathbb{R}^{n}$, where each coordinate $h_{j}$ of $h$ gives the value of a lifting function on the point $a_{j}$ of $\mathcal{A}$. Denote by $\varphi_{\Delta, h}$ the unique affine function that agrees with $h$ on the points of $\Delta$, that is, $\varphi_{\Delta, h}\left(a_{j}\right)=h_{j}$ for all $a_{j} \in \Delta$. We associate with $\Delta$ the following cone:

$$
\mathcal{C}_{\Delta}=\left\{h=\left(h_{1}, \ldots, h_{n}\right) \in \mathbb{R}^{n}: \varphi_{\Delta, h}\left(a_{j}\right)<h_{j} \text { for all } a_{j} \notin \Delta\right\} .
$$

Assume that two simplices $\Delta_{1}$ and $\Delta_{2}$ share a common facet, that is, the points in $\mathcal{A}$ that lie in a face of dimension $d-1$ of its convex hull, and they only intersect there (see Figure 4 for an example). In this case, it can be shown that the cone $\mathcal{C}_{\Delta_{1}, \Delta_{2}}$ defined as the intersection

$$
\mathcal{C}_{\Delta_{1}, \Delta_{2}}=\mathcal{C}_{\Delta_{1}} \cap \mathcal{C}_{\Delta_{2}}
$$

is nonempty (see Proposition 2.5 in [4]).
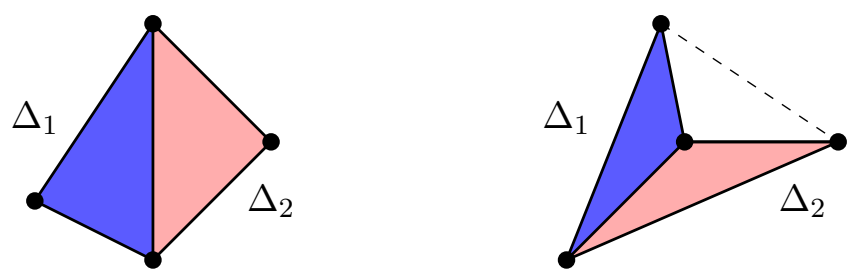

Figure 4 . Examples of simplices $\Delta_{1}$ and $\Delta_{2}$, which share a facet, $d=2$.

Example 2.5. (Example 2.1 and 2.4 continued). We compute the cone $\mathcal{C}_{\Delta_{1}, \Delta_{2}}$ for the simplices $\Delta_{1}$ and $\Delta_{2}$. We take any values $h_{1}, h_{2}, h_{3}$ corresponding to the points of $\Delta_{1}$ : $(0,0),(2,0)$ and $(0,1)$ respectively.

We consider then the unique affine linear function $\varphi_{\Delta_{1}, h}(x, y)$ which satisfies $\varphi_{\Delta_{1}, h}(0,0)=$ $h_{1}, \varphi_{\Delta_{1}, h}(2,0)=h_{2}$ and $\varphi_{\Delta_{1}, h}(0,1)=h_{3}$ :

$$
\varphi_{\Delta_{1}, h}(x, y)=\left(\frac{h_{2}-h_{1}}{2}\right) x+\left(h_{3}-h_{1}\right) y+h_{1} .
$$

We need that

$$
\begin{aligned}
& \varphi_{\Delta_{1}, h}(2,1)=-h_{1}+h_{2}+h_{3}<h_{4}, \quad \varphi_{\Delta_{1}, h}(1,2)=-\frac{3}{2} h_{1}+\frac{1}{2} h_{2}+2 h_{3}<h_{5}, \\
& \varphi_{\Delta_{1}, h}(1,3)=-\frac{5}{2} h_{1}+\frac{1}{2} h_{2}+3 h_{3}<h_{6} .
\end{aligned}
$$

Then, we have the description

$$
\begin{array}{r}
\mathcal{C}_{\Delta_{1}}=\left\{h=\left(h_{1}, \ldots, h_{6}\right) \in \mathbb{R}^{6}: h_{1}-h_{2}-h_{3}+h_{4}>0,3 h_{1}-h_{2}-4 h_{3}+2 h_{5}>0,\right. \\
\left.5 h_{1}-h_{2}-6 h_{3}+2 h_{6}>0\right\} .
\end{array}
$$

In analogous way we can compute $\mathcal{C}_{\Delta_{2}}$ :

$$
\begin{array}{r}
\mathcal{C}_{\Delta_{2}}=\left\{h=\left(h_{1}, \ldots, h_{6}\right) \in \mathbb{R}^{6}: h_{1}-h_{2}-h_{3}+h_{4}>0,2 h_{2}-h_{3}-3 h_{4}+2 h_{5}>0,\right. \\
\left.4 h_{2}-h_{3}-5 h_{4}+2 h_{6}>0\right\} .
\end{array}
$$

We observe that one of the inequalities appears twice, then $\mathcal{C}_{\Delta_{1}, \Delta_{2}}$ is defined by five inequalities. We can write:

$$
\mathcal{C}_{\Delta_{1}, \Delta_{2}}=\left\{h=\left(h_{1}, \ldots, h_{6}\right) \in \mathbb{R}^{6}:\left\langle m_{r}, h\right\rangle>0, r=1, \ldots, 5\right\},
$$

where $m_{1}=(1,-1,-1,1,0,0), m_{2}=(3,-1,-4,0,2,0), m_{3}=(5,-1,-6,0,0,2), m_{4}=$ $(0,2,-1,-3,2,0), m_{5}=(0,4,-1,-5,0,2)$, and $\langle$,$\rangle denotes the canonical inner product.$

Remark 2.6. Take any vector $h \in \mathbb{R}^{n}$ and consider the lower convex hull $\mathcal{L}$ of the $n$ lifted points $\left(a_{j}, h_{j}\right) \in \mathbb{R}^{d+1}$, with $a_{j}, j=1, \ldots, n$, in the support $\mathcal{A}$ (see Figure 5 ). Project to $\mathbb{R}^{d}$ the subsets of points in each of the faces of $\mathcal{L}$. These subsets define a regular subdivision of $\mathcal{A}$ associated with $h$. When the lifting vector $h$ is generic, the regular subdivision is a 
regular triangulation, in which all the subsets are simplices. Note that given a simplex $\Delta \subset \mathcal{A}$, the cone $\mathcal{C}_{\Delta}$ consists of the vectors $h$ for which the induced regular subdivision contains the simplex $\Delta$.

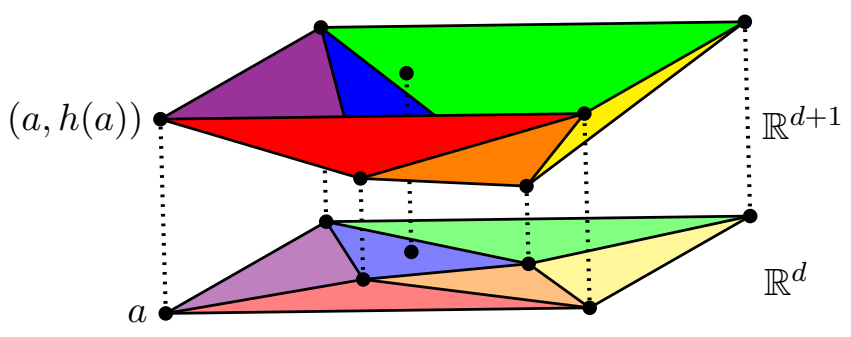

Figure 5. Regular triangulation.

Given any $h \in \mathbb{R}^{n}$, consider the following family of polynomial systems parametrized by a positive real number $t$, which coincides for $t=1$ with the system defined by the polynomials in (2.1):

$$
f_{1, t}(x)=\cdots=f_{d, t}(x)=0,
$$

where

$$
f_{i, t}(x)=\sum_{j=1}^{n} c_{i j} t^{h_{j}} x^{a_{j}} \in \mathbb{R}\left[x_{1}, \ldots, x_{d}\right], i=1, \ldots, d .
$$

For each positive real value of $t$, this system has again support included in $\mathcal{A}$. Recall that a common root of (2.5) is nondegenerate when it is not a zero of the Jacobian of $f_{1, t}, \ldots, f_{d, t}$. The following result is a particular case of Theorem 3.4 in [5].

Theorem 2.7. Let $\mathcal{A}=\left\{a_{1}, \ldots, a_{n}\right\} \subset \mathbb{Z}^{d}$ be a finite point configuration and $C=\left(c_{i j}\right) \in$ $\mathbb{R}^{d \times n}$ a matrix. Let $\Delta_{1}, \Delta_{2}$ be two d-simplices with vertices in $\mathcal{A}$ which share a facet, and which are positively decorated by the matrix $C$. Let $h$ be any vector in the cone $\mathcal{C}_{\Delta_{1}, \Delta_{2}}$. Then, there exists $t_{0} \in \mathbb{R}_{>0}$ such that for all $0<t<t_{0}$, the number of (nondegenerate) solutions of (2.5) contained in the positive orthant is at least two.

Example 2.8. (Example 2.1, 2.4 and 2.5 continued). The simplices $\Delta_{1}$ and $\Delta_{2}$ are positively decorated by $C$ and share a facet. Then, if we take $h \in \mathcal{C}_{\Delta_{1}, \Delta_{2}}$, there exists $t_{0} \in \mathbb{R}_{>0}$ such that for all $0<t<t_{0}$, the number of (nondegenerate) solutions of the deformed system

$$
\begin{aligned}
t^{h_{1}}-t^{h_{2}} 2 x^{2}+t^{h_{3}} y+t^{h_{4}} x^{2} y-t^{h_{5}} x y^{2} & =0, \\
-t^{h_{1}} 2+t^{h_{2}} x^{2}-t^{h_{4}} x^{2} y-t^{h_{5}} x y^{2}+t^{h_{6}} x y^{3} & =0,
\end{aligned}
$$

is at least two.

Indeed, it is easy to check that the simplices $\Delta_{4}=\{(0,1),(1,2),(1,3)\}$ and $\Delta_{5}=$ $\{(2,1),(1,2),(1,3)\}$ in Figure 6 are also positively decorated by the matrix $C$. In fact, by Theorem 2.9 in [4], for any $h \in \mathcal{C}_{\Delta_{1}} \cap \mathcal{C}_{\Delta_{2}} \cap \mathcal{C}_{\Delta_{4}} \cap \mathcal{C}_{\Delta_{5}}$ there exists $t_{0}$ such that if $0<t<t_{0}$ the system (2.6) has at least four positive solutions. Here four is the number of simplices which are positively decorated. For example if we take $h_{1}=1, h_{2}=h_{3}=h_{4}=0, h_{5}=1$ and $h_{6}=3$, we obtain the regular triangulation in Figure 6 , and if we choose $t=1 / 12$, system (2.5) has four positive solutions. This fact can be checked using the free Computer Algebra System Singular [13] with the library "signcond.lib" implemented by E. Tobis, with the following code:

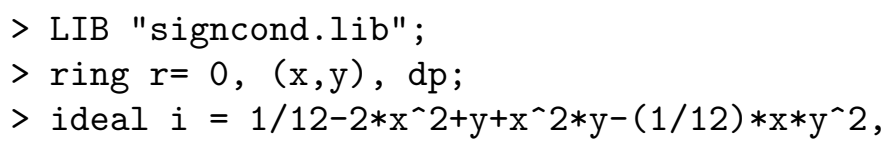




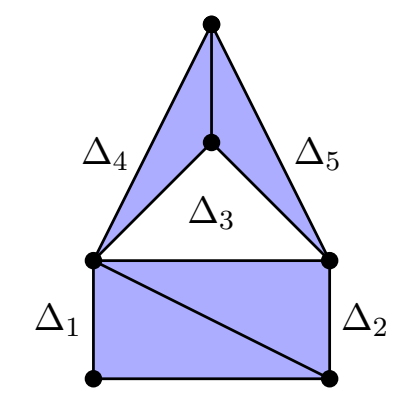

Figure 6. Simplices of $\mathcal{A}$ in Example 2.1, which are positively decorated by the matrix $C$.

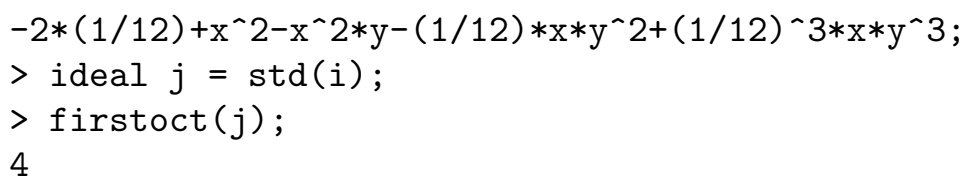

Note that this procedure is symbolic and thus certified, as opposed to numeric algorithms to compute the roots which can be affected by numerical unstability. It is based on the algorithms described in [2].

We now state a similar result, but here we describe a subset with nonempty interior in the space of coefficients where we can find at least two positive solutions of the associated system. This is a simplified version of Theorem 2.11 in [4].

Theorem 2.9. Consider a set $\mathcal{A}=\left\{a_{1}, \ldots, a_{n}\right\}$ of $n$ points in $\mathbb{R}^{d}$ and a matrix $C=$ $\left(c_{i, j}\right) \in \mathbb{R}^{d \times n}$. Assume there are two d-simplices $\Delta_{1}, \Delta_{2}$ with vertices in $\mathcal{A}$, which share a facet and are positively decorated by the matrix $C$. Assume that the cone $\mathcal{C}_{\Delta_{1}, \Delta_{2}}$ is defined by the inequalities

$$
\left\langle m_{r}, h\right\rangle>0, r=1, \ldots, \ell,
$$

where $m_{r}=\left(m_{r, 1}, \ldots, m_{r, n}\right) \in \mathbb{R}^{n}$.

Then, there exists constants $M_{1}, \ldots, M_{\ell}>0$ such that for any $\gamma$ in the open set

$$
U=\left\{\gamma \in \mathbb{R}_{>0}^{n}: \gamma^{m_{r}}<M_{r}, r=1 \ldots, \ell\right\},
$$

the system

$$
\sum_{j=1}^{n} c_{i j} \gamma_{j} x^{a_{j}}=0, \quad i=1, \ldots, d,
$$

has at least 2 nondegenerate solutions in the positive orthant.

Remark 2.10. Note that the choice of the positive constants $M_{1}, \ldots, M_{\ell}$ is not algorithmic, but we describe an open set in coefficient space for which more than one positive solution can be found. Furthermore, inequalities (2.7) indicate how to scale the coefficients of the system in order to get at least two positive solutions.

\section{ENZyMatic CASCADES WITH TWO LAYERS}

In this section we work with the case of an enzymatic cascade with two layers, and then in Section 4 we will work with the general case. The network involves two phosphorylation cycles. We call $S_{1}$ and $S_{2}$ the substrate proteins in the first and second layers respectively. The upper index can be interpreted as the absence (0) or the presence (1) of a phosphate group. The phosphorylation in the first layer is catalyzed by the enzyme $E$. The activated protein $S_{1}^{1}$ in the first layer acts as the modifier enzyme in the second layer, which is depicted in (A) in Figure 2.

Note that the dephosphorylation is carried out by the same phosphatase $F$, which as we pointed out in the Introduction gives the capacity for multistationarity to the network 
by [15]. The kinetics of this network is deduced by applying the law of mass-action to the following labeled digraph:

$$
\begin{aligned}
& S_{1}^{0}+E \underset{k_{\mathrm{off}_{1}}^{\rightleftarrows}}{\stackrel{k_{\mathrm{on}_{1}}}{\rightleftarrows}} Y_{1}^{0} \stackrel{k_{\mathrm{cat}_{1}}}{\rightarrow} S_{1}^{1}+E \quad S_{2}^{0}+S_{1}^{1} \underset{k_{\mathrm{off}_{2}}}{\stackrel{k_{\mathrm{on}_{2}}}{\rightleftarrows}} Y_{2}^{0} \stackrel{k_{\mathrm{cat}_{2}}}{\rightarrow} S_{2}^{1}+S_{1}^{1} \\
& S_{1}^{1}+F \underset{\ell_{\mathrm{off}_{1}}}{\stackrel{\ell_{\mathrm{on}_{1}}}{\rightleftarrows}} Y_{1}^{1} \stackrel{\ell_{\mathrm{cat}}}{\rightarrow} S_{1}^{0}+F \quad S_{2}^{1}+F \underset{\ell_{\mathrm{off}_{2}}}{\stackrel{\ell_{\mathrm{on}_{2}}}{\rightleftarrows}} Y_{2}^{1} \stackrel{\ell_{\mathrm{cat}_{2}}}{\rightarrow} S_{2}^{0}+F .
\end{aligned}
$$

We denote by $Y_{1}^{0}, Y_{2}^{0}, Y_{1}^{1}, Y_{2}^{1}$ the intermediate complexes, which consist of a single chemical species formed by the union of the substrate with the enzyme. The concentrations of the species will be denoted with small letters, for example $s_{1}^{0}$ will denote the concentration of $S_{1}^{0}$. The associated dynamical system that arises under mass-action kinetics equals:

$$
\begin{aligned}
\frac{d s_{1}^{0}}{d t} & =-k_{\mathrm{on}_{1}} s_{1}^{0} e+k_{\mathrm{off}_{1}} y_{1}^{0}+\ell_{\mathrm{cat}_{1}} y_{1}^{1}, & \frac{d y_{1}^{1}}{d t} & =\ell_{\mathrm{on}_{1}} s_{1}^{1} f-\left(\ell_{\mathrm{off}_{1}}+\ell_{\mathrm{cat}_{1}}\right) y_{1}^{1}, \\
\frac{d s_{1}^{1}}{d t} & =k_{\mathrm{cat}_{1}} y_{1}^{0}-\ell_{\mathrm{on}_{1}} s_{1}^{1} f+\ell_{\mathrm{off}_{1}} y_{1}^{1} & \frac{d y_{2}^{0}}{d t} & =k_{\mathrm{on}_{2}} s_{2}^{0} s_{1}^{1}-\left(k_{\mathrm{off}_{2}}+k_{\mathrm{cat}_{2}}\right) y_{2}^{0}, \\
& +k_{\mathrm{on}_{2}} s_{2}^{0} s_{1}^{1}+\left(k_{\mathrm{off}_{2}}+k_{\mathrm{cat}_{2}}\right) y_{2}^{0}, & \frac{d y_{2}^{1}}{d t} & =\ell_{\mathrm{on}_{2}} s_{2}^{1} f-\left(\ell_{\mathrm{off}_{2}}+\ell_{\mathrm{cat}_{2}}\right) y_{2}^{1}, \\
\frac{d s_{2}^{0}}{d t} & =-k_{\mathrm{on}_{2}} s_{2}^{0} s_{1}^{1}+k_{\mathrm{off}_{2}} y_{2}^{0}+\ell_{\mathrm{cat}_{2}} y_{2}^{1}, & \frac{d e}{d t} & =-k_{\mathrm{on}_{1}} s_{1}^{0} e+\left(k_{\mathrm{off}_{1}}+k_{\mathrm{cat}_{1}}\right) y_{1}^{0}, \\
\frac{d s_{2}^{1}}{d t} & =k_{\mathrm{cat}_{2}} y_{2}^{0}-\ell_{\mathrm{on}_{2}} s_{2}^{1} f+\ell_{\mathrm{off}_{2}} y_{2}^{1}, & \frac{d f}{d t} & =-\ell_{\mathrm{on}_{1}} s_{1}^{1} f+\left(\ell_{\mathrm{off}_{1}}+\ell_{\mathrm{cat}_{1}}\right) y_{1}^{1} \\
\frac{d y_{1}^{0}}{d t} & =k_{\mathrm{on}_{1}} s_{1}^{0} e-\left(k_{\mathrm{off}_{1}}+k_{\mathrm{cat}_{1}}\right) y_{1}^{0}, & & -\ell_{\mathrm{on}_{2}} s_{2}^{1} f+\left(\ell_{\mathrm{off}_{2}}+\ell_{\mathrm{cat}_{2}}\right) y_{2}^{1} .
\end{aligned}
$$

In this case, there is a basis of the conservation laws given by the four linear equations:

$$
\begin{aligned}
e+y_{1}^{0} & =E_{t o t}, \\
f+y_{1}^{1}+y_{2}^{1} & =F_{t o t}, \\
s_{1}^{0}+s_{1}^{1}+y_{1}^{0}+y_{1}^{1}+y_{2}^{0} & =S_{1, t o t}, \\
s_{2}^{0}+s_{2}^{1}+y_{2}^{0}+y_{2}^{1} & =S_{2, t o t} .
\end{aligned}
$$

Enzymatic cascades are an example of s-toric MESSI networks, defined in [26]. By Theorem 3.5 in [26] we can find binomial equations that describe the steady states. This is a general procedure, that in this case is easily obtained by manipulating the differential equations. First, the concentrations of the intermediates species $y_{1}^{0}, y_{1}^{1}, y_{2}^{0}, y_{2}^{1}$ at steady state satisfy the following binomial equations:

$$
\begin{aligned}
y_{1}^{0}-K_{1} e s_{1}^{0}=0, & y_{1}^{1}-L_{1} f s_{1}^{1}=0, \\
y_{2}^{0}-K_{2} s_{1}^{1} s_{2}^{0}=0, & y_{2}^{1}-L_{2} f s_{2}^{1}=0,
\end{aligned}
$$

where $K_{1}=\frac{k_{\mathrm{on}_{1}}}{k_{\mathrm{off}_{1}}+k_{\mathrm{cat}_{1}}}, K_{2}=\frac{k_{\mathrm{on}_{2}}}{k_{\mathrm{off}_{2}}+k_{\mathrm{cat}_{2}}}, L_{1}=\frac{\ell_{\mathrm{on}_{1}}}{\ell_{\mathrm{off}_{1}}+\ell_{\mathrm{cat}_{1}}}$ and $L_{2}=\frac{\ell_{\mathrm{on}_{2}}}{\ell_{\mathrm{off}_{2}}+\ell_{\mathrm{cat}_{2}}}\left(K_{1}^{-1}, K_{2}^{-1}\right.$, $L_{1}^{-1}$ and $L_{2}^{-1}$ are usually called Michaelis-Menten constants). The whole steady state variety can be cut out in the positive orthant by adding to the binomials in (3.3), the following binomial equations:

$$
\tau_{1} s_{1}^{0} e-\nu_{1} s_{1}^{1} f=0, \quad \tau_{2} s_{2}^{0} s_{1}^{1}-\nu_{2} s_{2}^{1} f=0,
$$

where $\tau_{1}=k_{\text {cat }_{1}} K_{1}, \tau_{2}=k_{\text {cat }_{2}} K_{2}, \nu_{1}=\ell_{\text {cat }_{1}} L_{1}$ and $\nu_{2}=\ell_{\text {cat }_{2}} L_{2}$.

Therefore, we can parametrize the positive steady states by monomials. For instance, we can write the concentration at steady state of $s_{1}^{0}, s_{2}^{0}$ and the intermediate species, in 
terms of the species $\left(e, f, s_{1}^{1}, s_{2}^{1}\right)$ :

$$
\begin{array}{lll}
s_{1}^{0}=G_{1} \frac{s_{1}^{1} f}{e}, & y_{1}^{0}=K_{1} G_{1} s_{1}^{1} f, & y_{1}^{1}=L_{1} s_{1}^{1} f, \\
s_{2}^{0}=G_{2} \frac{s_{2}^{1} f}{s_{1}^{1}}, & y_{2}^{0}=K_{2} G_{2} s_{2}^{1} f, & y_{2}^{1}=L_{2} s_{2}^{1} f,
\end{array}
$$

where $G_{1}=\frac{\nu_{1}}{\tau_{1}}$ and $G_{2}=\frac{\nu_{2}}{\tau_{2}}$.

Now, we apply our results to this case. Denote by

$$
A_{1}=\frac{\ell_{\mathrm{cat}_{1}}}{k_{\mathrm{cat}_{1}}}, \quad A_{2}=\frac{\ell_{\mathrm{cat}_{2}}}{k_{\mathrm{cat}_{2}}}
$$

and assume that $S_{1, t o t}, S_{2, t o t}, E_{t o t}, F_{t o t}>0$. Consider the following rational functions $\alpha_{1}, \alpha_{2}, \alpha_{3}, \alpha_{4}$ depending on the catalytic reaction rate constants and total concentration constants:

$$
\begin{aligned}
& \alpha_{1}=\frac{S_{1, t o t}}{F_{t o t}}-A_{2}, \\
& \alpha_{2}=\left(A_{1}+1\right)-\frac{S_{1, t o t}}{F_{t o t}}, \\
& \alpha_{3}=\frac{A_{1}+1-A_{2}}{A_{1}} \frac{E_{t o t}}{F_{t o t}}-\left(\frac{S_{1, t o t}}{F_{t o t}}-A_{2}\right), \\
& \alpha_{4}=\frac{A_{1}+1-A_{2}}{A_{2}+1} \frac{S_{2, t o t}}{F_{t o t}}-\left(A_{1}+1-\frac{S_{1, t o t}}{F_{t o t}}\right) .
\end{aligned}
$$

We then have:

Theorem 3.1. Consider the enzymatic cascade with two layers with digraph as in (3.1) and let $A_{1}, A_{2}$ as in (3.5). Assume that the reaction rate constants verify $A_{1}+1>A_{2}$ and the total concentration constants verify the inequalities $\alpha_{1}, \alpha_{2}, \alpha_{3}, \alpha_{4}>0$, that is:

$$
\begin{aligned}
A_{1}+1> & \frac{S_{1, t o t}}{F_{t o t}}>A_{2}, \quad \frac{E_{t o t}}{F_{t o t}}>\left(\frac{S_{1, t o t}}{F_{t o t}}-A_{2}\right) \frac{A_{1}}{A_{1}+1-A_{2}}, \\
& \frac{S_{2, t o t}}{F_{t o t}}>\left(A_{1}+1-\frac{S_{1, t o t}}{F_{t o t}}\right) \frac{A_{2}+1}{A_{1}+1-A_{2}},
\end{aligned}
$$

or instead, that $A_{1}+1<A_{2}$ and $\alpha_{1}, \alpha_{2}, \alpha_{3}, \alpha_{4}<0$.

Fix generic positive numbers $h_{2}, h_{3}, h_{7}, h_{8}$ such that $h_{8}<h_{2}$. Then, there exists $t_{0}>0$ such that for any value of $t \in\left(0, t_{0}\right)$ the system has at least two positive steady states after modifying the coefficients $k_{\mathrm{on}_{1}}, k_{\mathrm{on}_{2}}, \ell_{\mathrm{on}_{1}}, \ell_{\mathrm{on}_{2}}$ via the rescaling $t^{-h_{7}} k_{\mathrm{on}_{1}}, t^{-h_{3}-h_{8}} k_{\mathrm{on}_{2}}$, $t^{-h_{2}-h_{3}} \ell_{\mathrm{on}_{1}}$ and $t^{-h_{2}} \ell_{\mathrm{on}_{2}}$.

Also, for any fixed choice of reaction rate constants and total concentration constants lying in the open set defined by one of the previous set of inequalities, there exist positive constants $M_{1}, \ldots, M_{6}$ such that for any values of $\beta_{1}, \beta_{2}, \eta_{1}, \eta_{2}$ verifying

$$
\frac{1}{\eta_{2}}<M_{1}, \quad \frac{\eta_{2}}{\eta_{1}}<M_{2}, \quad \frac{1}{\beta_{1}}<M_{3}, \quad \frac{\eta_{1}}{\eta_{2} \beta_{2}}<M_{4}, \quad \frac{\beta_{2}}{\eta_{1}}<M_{5}, \quad \frac{1}{\beta_{2}}<M_{6},
$$

the rescaling of the given parameters $k_{\mathrm{on}_{0}}, k_{\mathrm{on}_{1}}, \ell_{\mathrm{on}_{0}}$ and $\ell_{\mathrm{on}_{1}}$ by $\beta_{1} k_{\mathrm{on}_{1}}, \beta_{2} k_{\mathrm{on}_{2}}, \eta_{1} \ell_{\mathrm{on}_{1}}$ and $\eta_{2} \ell_{\mathrm{on}_{2}}$ respectively, gives raise to a multistationary system.

Proof. We substitute the monomial parametrization in (3.4) of the steady states in terms of the concentrations of the species $e, f, s_{1}^{1}, s_{2}^{1}$ into the linear conservation relations (3.2). We write this system in matricial form:

$$
C\left(e \quad f \quad s_{1}^{1} \quad s_{2}^{1} \quad s_{1}^{1} f \quad s_{2}^{1} f \quad s_{1}^{1} f e^{-1} \quad s_{2}^{1} f\left(s_{1}^{1}\right)^{-1} \quad 1\right)^{t}=0,
$$


where the matrix $C \in \mathbb{R}^{4 \times 9}$ of coefficients equals:

$$
C=\left(\begin{array}{ccccccccc}
1 & 0 & 0 & 0 & K_{1} G_{1} & 0 & 0 & 0 & -E_{t o t} \\
0 & 1 & 0 & 0 & L_{1} & L_{2} & 0 & 0 & -F_{t o t} \\
0 & 0 & 1 & 0 & K_{1} G_{1}+L_{1} & K_{2} G_{2} & G_{1} & 0 & -S_{1, t o t} \\
0 & 0 & 0 & 1 & 0 & K_{2} G_{2}+L_{2} & 0 & G_{2} & -S_{2, t o t}
\end{array}\right) .
$$

If we order the variables as before, the support of this system is:

$$
\begin{array}{r}
\mathcal{A}=\{(1,0,0,0),(0,1,0,0),(0,0,1,0),(0,0,0,1),(0,1,1,0), \\
(0,1,0,1),(-1,1,1,0),(0,1,-1,1),(0,0,0,0)\} .
\end{array}
$$

We want to find two positively decorated 4 -simplices with vertices in $\mathcal{A}$ which share one facet. For example we take the simplices

$$
\begin{aligned}
& \Delta_{1}=\{(1,0,0,0),(0,0,0,1),(0,1,1,0),(0,1,0,1),(0,0,0,0)\}, \\
& \Delta_{2}=\{(1,0,0,0),(0,1,1,0),(0,1,0,1),(0,1,-1,1),(0,0,0,0)\} .
\end{aligned}
$$

It is straightforward to check that both simplices are positively decorated by $C$ if either $A_{1}+1>A_{2}$ and $\alpha_{1}, \alpha_{2}, \alpha_{3}, \alpha_{4}>0$, or $A_{1}+1<A_{2}$ and $\alpha_{1}, \alpha_{2}, \alpha_{3}, \alpha_{4}<0$, as in the statement.

Given $h \in \mathcal{C}_{\Delta_{1}, \Delta_{2}}$, by Theorem 2.7, there exists $t_{0} \in \mathbb{R}_{+}$such that for all $0<t<t_{0}$, the number of positive (nondegenerate) solutions of the scaled system:

$$
\begin{aligned}
t^{h_{1}} e+t^{h_{5}} K_{1} G_{1} s_{1}^{1} f-t^{h_{9}} E_{\text {tot }} & =0, \\
t^{h_{2}} f+t^{h_{5}} L_{1} f s_{1}^{1}+t^{h_{6}} L_{2} f s_{2}^{1}-t^{h_{9}} F_{t o t} & =0, \\
t^{h_{3}} s_{1}^{1}+t^{h_{7}} G_{1} \frac{s_{1}^{1} f}{e}+t^{h_{5}}\left(K_{1} G_{1}+L_{1}\right) s_{1}^{1} f+t^{h_{6}} K_{2} G_{2} s_{2}^{1} f-t^{h_{9}} S_{1, t o t} & =0, \\
t^{h_{4}} s_{2}^{1}+t^{h_{8}} G_{2} \frac{s_{2}^{1} f}{s_{1}^{1}}+t^{h_{6}}\left(K_{2} G_{2}+L_{2}\right) s_{2}^{1} f-t^{h_{9}} S_{2, t o t} & =0,
\end{aligned}
$$

is at least two. If we think of the vector $h$ as a function $\mathcal{A} \rightarrow \mathbb{R}$ (defined by $h\left(a_{j}\right)=h_{j}$ ), then $h_{1}=h(1,0,0,0), h_{2}=h(0,1,0,0), h_{3}=h(0,0,1,0), h_{4}=h(0,0,0,1), h_{5}=h(0,1,1,0)$, $h_{6}=h(0,1,0,1), h_{7}=h(-1,1,1,0), h_{8}=h(0,1,-1,1)$ and $h_{9}=h(0,0,0,0)$. Let $\varphi_{1}$ and $\varphi_{2}$ be the affine linear functions which agree with $h$ on the simplices $\Delta_{1}$ and $\Delta_{2}$ respectively. We can take $h_{1}=h_{4}=h_{5}=h_{6}=h_{9}=0$. Then $\varphi_{1}=0, h_{8}>0$ and $\varphi_{2}$ is defined by $\varphi_{2}(x, y, z, w)=h_{8} y-h_{8} z-h_{8} w$. Moreover,

$$
\begin{aligned}
& 0<h_{2}, \quad \varphi_{2}(0,1,0,0)=h_{8}<h_{2}, \\
& 0<h_{3}, \quad \varphi_{2}(0,0,1,0)=-h_{8}<h_{3}, \\
& 0<h_{7}, \quad \varphi_{2}(-1,1,1,0)=0<h_{7},
\end{aligned}
$$

where we could take $h_{2}, h_{3}$ and $h_{7}$ generic.

If we change the variables $\bar{f}=t^{h_{2}} f, \bar{s}_{1}^{1}=t^{h_{3}} s_{1}^{1}$, we get the following (Laurent) polynomial equations:

$$
\begin{aligned}
e+t^{-h_{2}-h_{3}} K_{1} G_{1} \bar{s}_{1}^{1} \bar{f}-E_{t o t} & =0, \\
\bar{f}+t^{-h_{2}-h_{3}} L_{1} \bar{f} \bar{s}_{1}^{1}+t^{-h_{2}} L_{2} \bar{f} s_{2}^{1}-F_{t o t} & =0, \\
\bar{s}_{1}^{1}+t^{h_{7}-h_{2}-h_{3}} G_{1} \frac{\bar{s}_{1}^{1} \bar{f}}{e}+t^{-h_{2}-h_{3}}\left(K_{1} G_{1}+L_{1}\right) \bar{s}_{1}^{1} \bar{f}+t^{-h_{2}} K_{2} G_{2} s_{2}^{1} \bar{f}-S_{1, t o t}= & 0, \\
s_{2}^{1}+t^{h_{8}+h_{3}-h_{2}} G_{2} \frac{s_{2}^{1} \bar{f}}{\bar{s}_{1}^{1}}+t^{-h_{2}}\left(K_{2} G_{2}+L_{2}\right) s_{2}^{1} \bar{f}-S_{2, t o t} & =0 .
\end{aligned}
$$

It is straightforward to verify that if we scale the constants:

$$
t^{-h_{7}} K_{1}, t^{-h_{3}-h_{8}} K_{2}, t^{-h_{2}-h_{3}} L_{1}, t^{-h_{2}} L_{2},
$$

and we keep fixed the values of $k_{\mathrm{cat}_{1}}, k_{\mathrm{cat}_{2}}, \ell_{\mathrm{cat}_{1}}$ and $\ell_{\mathrm{cat}_{2}}$ and the total values $E_{\text {tot }}, F_{\text {tot }}$, $S_{1, t o t}$ and $S_{2, t o t}$, the intersection of the steady state variety and the linear varieties of fixed total concentrations of the dynamical system associated with the corresponding network, is described by system (3.9). 
It is easy to check that to get the scaling in (3.10), it is enough to rescale the original constants as follows: $t^{-h_{7}} k_{\mathrm{on}_{1}}, t^{-h_{3}-h_{8}} k_{\mathrm{on}_{2}}, t^{-h_{2}-h_{3}} \ell_{\mathrm{on}_{1}}$ and $t^{-h_{2}} \ell_{\mathrm{on}_{2}}$. Then, for these choices of constants the system has at least two positive steady states. The last part of the statement follows from the previous rescaling or from the inequalities that define the cone $\mathcal{C}_{\Delta_{1}, \Delta_{2}}$ of heights inducing regular subdivisions of the convex hull of $\mathcal{A}$ that contain $\Delta_{1}$ and $\Delta_{2}$ and Theorem 2.9. For instance, we can check that $\mathcal{C}_{\Delta_{1}, \Delta_{2}}$ is defined by 6 inequalities. We can write:

$$
\mathcal{C}_{\Delta_{1}, \Delta_{2}}=\left\{h=\left(h_{1}, \ldots, h_{8}\right) \in \mathbb{R}^{8}:\left\langle m_{r}, h\right\rangle>0, r=1, \ldots, 6\right\},
$$

where $\langle$,$\rangle denotes the canonical inner product of \mathbb{R}^{8}$ and $m_{1}=(0,1,0,1,0,-1,0,0,-1)$, $m_{2}=(0,0,1,-1,-1,1,0,0,0), m_{3}=(1,0,0,0,-1,0,1,0,-1), m_{4}=(0,0,0,1,1,-2,0,1,-1)$, $m_{5}=(0,1,0,0,-1,1,0,-1,0), m_{6}=(0,0,1,0,0,-1,0,1,-1)$. By Theorem 2.9 , there exist $M_{1}, \ldots, M_{6}>0$ such that for any $\gamma=\left(\gamma_{1}, \ldots, \gamma_{9}\right)$ in the open set

$$
U=\left\{\gamma \in \mathbb{R}_{>0}^{9}: \gamma^{m_{r}}<M_{r}, r=1 \ldots, 6\right\},
$$

the system

$$
\begin{aligned}
\gamma_{1} e+\gamma_{5} K_{1} G_{1} s_{1}^{1} f-\gamma_{9} E_{t o t} & =0, \\
\gamma_{2} f+\gamma_{5} L_{1} f s_{1}^{1}+\gamma_{6} L_{2} f s_{2}^{1}-\gamma_{9} F_{t o t}= & 0, \\
\gamma_{3} s_{1}^{1}+\gamma_{7} G_{1} \frac{s_{1}^{1} f}{e}+\gamma_{5}\left(K_{1} G_{1}+L_{1}\right) s_{1}^{1} f+\gamma_{6} K_{2} G_{2} s_{2}^{1} f-\gamma_{9} S_{1, t o t}= & 0, \\
\gamma_{4} s_{2}^{1}+\gamma_{8} G_{2} \frac{s_{2}^{1} f}{s_{1}^{1}}+\gamma_{6}\left(K_{2} G_{2}+L_{2}\right) s_{2}^{1} f-\gamma_{9} S_{2, t o t}= & 0,
\end{aligned}
$$

has at least two positive solutions. If we take $\gamma_{1}=\gamma_{2}=\gamma_{3}=\gamma_{4}=\gamma_{9}=1$, and we denote $\beta_{1}=\frac{\gamma_{5}}{\gamma_{7}}, \beta_{2}=\frac{\gamma_{6}}{\gamma_{8}}, \eta_{1}=\gamma_{5}$ and $\eta_{2}=\gamma_{6}$, the conditions such that $\gamma$ belongs to $U$ are equivalent to the conditions (3.6), and it is easy to check that the steady state equations of the network after the rescaling of the given parameters $k_{\mathrm{on}_{0}}, k_{\mathrm{on}_{1}}, \ell_{\mathrm{on}_{0}}$ and $\ell_{\mathrm{on}_{1}}$ by $\beta_{1} k_{\mathrm{on}_{1}}, \beta_{2} k_{\mathrm{on}_{2}}, \eta_{1} \ell_{\mathrm{on}_{1}}$ and $\eta_{2} \ell_{\mathrm{on}_{2}}$ give system (3.11).

Example 3.2. Note that the inequalities in the statement of Theorem 3.1 are clearly compatible. For example, the inequalities are satisfied if we take in the first case $\frac{\ell_{\text {cat }_{1}}}{k_{\text {cat }_{1}}}=1$, $\frac{\ell_{\text {cat }_{2}}}{k_{\text {cat }_{2}}}=1, E_{t o t}=F_{t o t}=20, S_{1, t o t}=S_{2, t o t}=30$. We can obtain in this case a value of $t$ such that the system (3.9) has two or more positive solutions, using Singular. Fix for example, $h_{2}=2, h_{3}=1, h_{7}=1, h_{8}=1, K_{1}=1, K_{2}=1, L_{1}=1$ and $L_{2}=1$. We have then that $G_{1}=1$ and $G_{2}=1$. If we take $t=\frac{1}{24}$, we have that the system has 3 positive solutions:

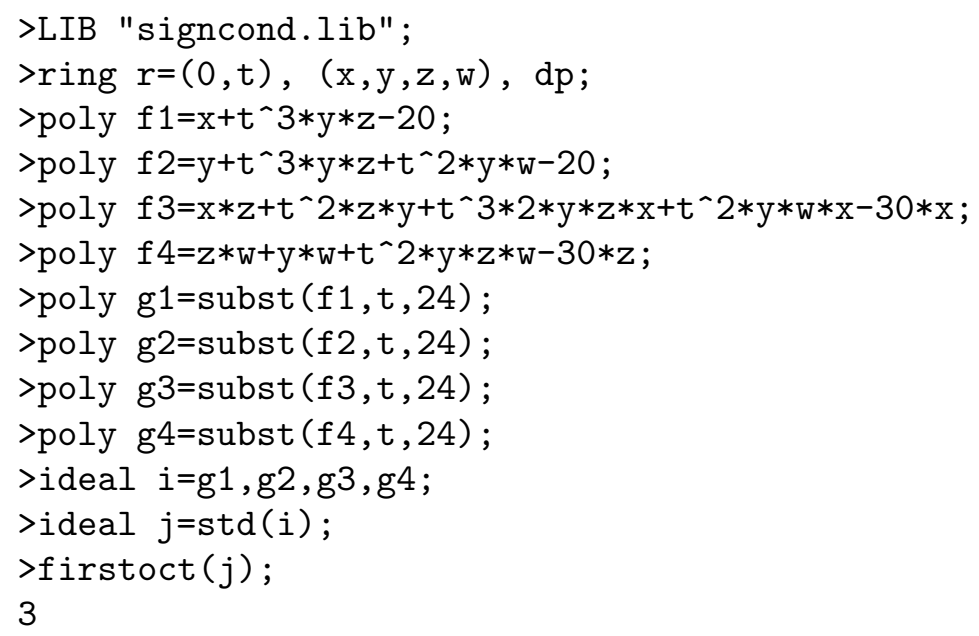

Here $x=e, y=\bar{f}, z=\bar{s}_{1}^{1}$ and $w=s_{2}^{1}$. It can be checked that if we take a slighly higher value $t=\frac{1}{23}$, the corresponding system has only one positive solution. 


\section{ENZYMATIC CASCADES WITH $n$ LAYERS}

We now present our results to the general case of an enzymatic cascade of $n$ layers, where we have $n$ phosphorylation cycles (as in Figure 7), under the assumption that there are (at least) two layers which share a phosphatase. We separate our study into two cases: the case of the occurrence of the same phosphatase in two consecutive layers (see Theorem 4.1) and the case where the layers which share the phosphatase are not consecutive (see Theorem 4.3). As we pointed out in the Introduction, the difficulty to deal with these networks is that the simplified polynomials that we get to describe the steady states in a given stoichiometric compatibility class depend on a number of variables that grows linearly with $n$ and the corresponding coefficient matrix does not have generic entries. We are nevertheless able to detect two simplices in these high dimensional spaces which share a facet, which are positively decorated by the (huge) coefficient matrix.

We first set the notation.

4.1. Our setting. Using the notation in Figure 7, we call $S_{i}^{0}, S_{i}^{1}$ the substrate proteins in the $i$-th layer, for $i=1, \ldots, n$. As before, the upper index can be interpreted as the absence (0) or the presence (1) of a phosphate group in the substrate. The phosphorylation in the first layer is catalyzed by the enzyme $S_{0}^{1}$. The activated protein $S_{i}^{1}$ in the $i$-th layer acts as the modifier enzyme in the $(i+1)$-th layer. The dephosphorylation in the $i$-th layer is carried out by a phosphatase $F_{i}$. Some of the $F_{i}$ can be the same species, that is, the same phosphatase can react at different layers.

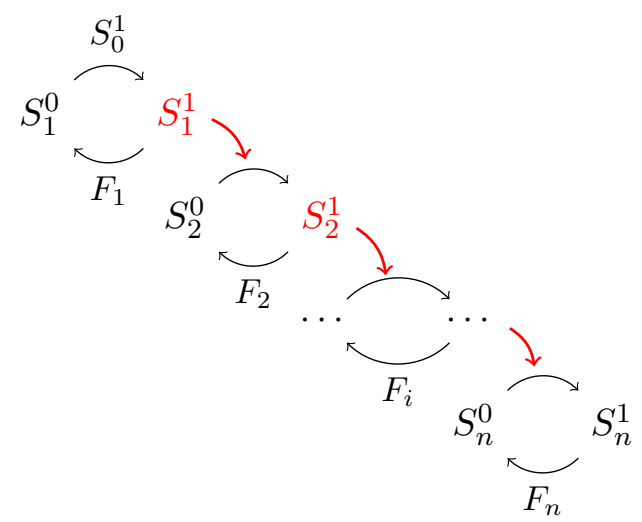

FiguRE 7. Enzymatic cascade with $n$ layers.

We assume the following reaction scheme:

$$
\begin{aligned}
& S_{i}^{0}+S_{i-1} \stackrel{k_{\mathrm{On}_{\mathrm{i}}}}{\rightleftarrows} Y_{i}^{0} \stackrel{k_{\mathrm{off}_{\mathrm{i}}}}{\rightarrow} S_{i}+S_{i-1}^{1}, \quad i=1 \ldots, n, \\
& S_{i}^{1}+F_{i} \underset{\ell_{\mathrm{off}_{\mathrm{i}}}}{\stackrel{\ell_{\mathrm{on}_{\mathrm{i}}}}{\leftrightarrows}} Y_{i}^{1} \stackrel{\ell_{\mathrm{cat}_{\mathrm{i}}}}{\rightarrow} S_{i}^{0}+F_{i}, \quad i=1, \ldots, n .
\end{aligned}
$$

We denote by $\mathcal{F}=\left\{P_{1}, \ldots, P_{r}\right\}$ the set of phosphatases that appear in the network. In this case we have $4 n+r+1$ chemical species: $S_{0}^{1}, S_{1}^{0}, S_{1}^{1}, S_{2}^{0}, S_{2}^{1}, \ldots, S_{n}^{0}, S_{n}^{1}, P_{1}$, $P_{2} \ldots P_{r}, Y_{1}^{0}, Y_{1}^{1}, Y_{2}^{0}, Y_{2}^{1}, \ldots, Y_{n}^{0}, Y_{n}^{1}$. We denote the concentration of the species with small letters. For each $j=1, \ldots, r$, we call $\Lambda_{j}=\left\{i \in\{1, \ldots, n\}: F_{i}=P_{j}\right\}$ and we consider the function $j:\{1, \ldots, n\} \rightarrow\{1, \ldots, r\}$, defined by $j(i)=j$ if $F_{i}=P_{j}$.

The associated dynamical system that arises under mass-action kinetics is equal to: 


$$
\begin{aligned}
& \frac{d s_{i}^{0}}{d t}=-k_{\mathrm{on}_{\mathrm{i}}} s_{i}^{0} s_{i-1}^{1}+k_{\mathrm{off}_{\mathrm{i}}} y_{i}^{0}+\ell_{\mathrm{cat}_{\mathrm{i}}} y_{i}^{1}, \quad i=1, \ldots, n, \\
& \frac{d s_{i}^{1}}{d t}=k_{\mathrm{cat}_{\mathrm{i}}} y_{i}^{0}-\ell_{\mathrm{on}_{\mathrm{i}}} s_{i}^{1} p_{j(i)}+\ell_{\mathrm{off}_{1}} y_{i}^{1}-k_{\mathrm{on}_{\mathrm{i}+1}} s_{i+1}^{0} s_{i}^{1}+\left(k_{\mathrm{off}_{\mathrm{i}+1}}+k_{\mathrm{cat}_{\mathrm{i}+1}}\right) y_{i+1}^{0}, \quad i=1, \ldots, n-1, \\
& \frac{d s_{n}^{1}}{d t}=k_{\mathrm{cat}_{\mathrm{n}}} y_{n}^{0}-\ell_{\mathrm{on}_{\mathrm{n}}} s_{n}^{1} p_{j(n)}+\ell_{\mathrm{off}_{\mathrm{n}}} y_{n}^{1}, \\
& \frac{d y_{i}^{0}}{d t}=k_{\mathrm{on}_{\mathrm{i}}} s_{i}^{0} s_{i-1}^{1}-\left(k_{\mathrm{off}_{\mathrm{i}}}+k_{\mathrm{cat}_{\mathrm{i}}}\right) y_{i}^{0}, \quad i=1, \ldots, n, \\
& \frac{d y_{i}^{1}}{d t}=\ell_{\mathrm{on}_{\mathrm{i}}} s_{i}^{1} p_{j(i)}-\left(\ell_{\mathrm{off}_{\mathrm{i}}}+\ell_{\mathrm{cat}_{\mathrm{i}}}\right) y_{i}^{1}, \quad i=1, \ldots, n, \\
& \frac{d s_{0}^{1}}{d t}=-\frac{d y_{1}^{0}}{d t}, \quad \frac{d p_{j}}{d t}=-\sum_{i \in \Lambda_{j}} \frac{d y_{i}^{1}}{d t}, \quad j=1, \ldots, r .
\end{aligned}
$$

The space of linear forms yielding conservation laws has dimension $n+r+1$, and we consider the following $n+r+1$ linearly independent conservation relations:

$$
\begin{aligned}
s_{0}^{1}+y_{1}^{0} & =S_{0, t o t}, \\
s_{i}^{0}+s_{i}^{1}+y_{i}^{0}+y_{i}^{1}+y_{i+1}^{0} & =S_{i, t o t}, \quad i=1, \ldots, n-1, \\
s_{n}^{0}+s_{n}^{1}+y_{n}^{0}+y_{n}^{1} & =S_{n, t o t}, \\
p_{j}+\sum_{i \in \Lambda_{j}} y_{i}^{1} & =P_{j, t o t}, \quad j=1, \ldots, r .
\end{aligned}
$$

Again, following the general procedure described in [26], we can find binomial equations that describe the concentration of the species at steady state. The concentration of the intermediate species satisfy these binomial equations:

$$
y_{i}^{0}-K_{i} s_{i-1}^{1} s_{i}^{0}=0, \quad i=1 \ldots, n, \quad y_{i}^{1}-L_{i} p_{j(i)} s_{i}^{1}=0, \quad i=1 \ldots, n,
$$

where $K_{i}=\frac{k_{\mathrm{on}_{\mathrm{i}}}}{k_{\mathrm{off}_{\mathrm{i}}}+k_{\mathrm{cat}_{\mathrm{i}}}}, i=1, \ldots, n, L_{i}=\frac{\ell_{\mathrm{on}_{\mathrm{i}}}}{\ell_{\mathrm{off}_{\mathrm{i}}}+\ell_{\mathrm{cat}_{\mathrm{i}}}}, i=1, \ldots, n$. The remaining binomials can be (algorithmically) chosen to be:

$$
\tau_{i} s_{i}^{0} s_{i-1}^{1}-\nu_{i} s_{i}^{1} p_{j(i)}=0, i=1, \ldots, n,
$$

where $\tau_{i}=k_{\text {cat }_{\mathrm{i}}} K_{i}, \nu_{i}=\ell_{\mathrm{cat}_{\mathrm{i}}} L_{i}, i=1, \ldots, n$.

As in the previous case of two layers, we can parametrize the positive steady states by monomials. For instance, we can write the concentrations of all species in terms of $s_{i}^{1}$, for $i=0,1, \ldots, n$ and $p_{1}, \ldots, p_{r}$ :

$$
\begin{array}{rrl}
s_{i}^{0}= & G_{i} \frac{s_{i}^{1} p_{j(i)}}{s_{i-1}^{1}}, & i=1, \ldots, n, \\
y_{i}^{0}= & K_{i} G_{i} s_{i}^{1} p_{j(i)}, & i=1, \ldots, n, \\
y_{i}^{1}= & L_{i} s_{i}^{1} p_{j(i)}, & i=1, \ldots, n,
\end{array}
$$

where $G_{i}=\frac{\nu_{i}}{\tau_{i}}$ for all $i=1, \ldots, n$.

As in (3.5), we will denote for any $j=1, \ldots, n$ :

$$
A_{j}=\frac{\ell_{\mathrm{cat}_{\mathrm{j}}}}{k_{\mathrm{cat}_{\mathrm{j}}}}
$$

4.2. Statement of our main results. Suppose first that there are two consecutive layers $i_{0}, i_{0}+1,1 \leq i_{0} \leq n-1$, with the same phosphatase $F$, that is, $P_{j\left(i_{0}\right)}=P_{j\left(i_{0}+1\right)}$, and with no restriction in the other layers. Let $\alpha_{1, i_{0}}, \alpha_{2, i_{0}}, \alpha_{3, i_{0}}$ and $\alpha_{4, i_{0}}$ be as in the case $n=2$, 
but these constants correspond to the restriction to the two layers $i_{0}$ and $i_{0}+1$. That is:

$$
\begin{aligned}
& \alpha_{1, i_{0}}=\frac{S_{i_{0}, t o t}}{F_{t o t}}-A_{i_{0}+1}, \\
& \alpha_{2, i_{0}}=\left(A_{i_{0}}+1\right)-\frac{S_{i_{0}, t o t}}{F_{t o t}}, \\
& \alpha_{3, i_{0}}=\frac{A_{i_{0}}+1-A_{i_{0}+1}}{A_{i_{0}}} \frac{S_{i_{0}-1, t o t}}{F_{t o t}}-\left(\frac{S_{i_{0}, t o t}}{F_{t o t}}-A_{i_{0}+1}\right), \\
& \alpha_{4, i_{0}}=\frac{A_{i_{0}}+1-A_{i_{0}+1}}{A_{i_{0}+1}+1} \frac{S_{i_{0}+1, t o t}}{F_{t o t}}-\left(A_{i_{0}}+1-\frac{S_{i_{0}, t o t}}{F_{t o t}}\right),
\end{aligned}
$$

where the value of $E_{t o t}$ in the case $n=2$ now corresponds to the value $S_{i_{0}-1, t o t}$ and $F_{\text {tot }}=P_{j\left(i_{0}\right), t o t}=P_{j\left(i_{0}+1\right), t o t}$. We have the following result:

Theorem 4.1. Suppose $n \geq 3$, and suppose that there are two consecutive layers $i_{0}, i_{0}+1$, with $1 \leq i_{0} \leq n-1$, with the same phosphatase and with no restriction in the other layers. Let $A_{i_{0}}, A_{i_{0}+1}$ be as in (4.2). Assume that the reaction rate constants verify

$$
A_{i_{0}}+1>A_{i_{0}+1}
$$

and the total concentration constants verify the inequalities $\alpha_{1, i_{0}}, \alpha_{2, i_{0}}, \alpha_{3, i_{0}}, \alpha_{4, i_{0}}>0$, that is:

$$
\begin{gathered}
A_{i_{0}}+1>\frac{S_{i_{0}, t o t}}{F_{t o t}}>A_{i_{0}+1}, \quad \frac{S_{i_{0}-1, t o t}}{F_{t o t}}>\left(\frac{S_{i_{0}, t o t}}{F_{t o t}}-A_{i_{0}+1}\right) \frac{A_{i_{0}}}{A_{i_{0}}+1-A_{i_{0}+1}}, \\
\frac{S_{i_{0}+1, t o t}}{F_{\text {tot }}}>\left(A_{i_{0}}+1-\frac{S_{i_{0}, t o t}}{F_{\text {tot }}}\right) \frac{A_{i_{0}+1}+1}{A_{i_{0}}+1-A_{i_{0}+1}}
\end{gathered}
$$

or

$$
A_{i_{0}}+1<A_{i_{0}+1}, \alpha_{1, i_{0}}, \alpha_{2, i_{0}}, \alpha_{3, i_{0}}, \alpha_{4, i_{0}}<0 .
$$

Then, there exists a rescaling in the constants $k_{\text {on }_{i}}, i=1, \ldots, n$ and $\ell_{o n_{i}}, i=1, \ldots, n$, such that the system has at least two positive steady states.

We will give an explicit rescaling in the proof.

Remark 4.2. In the statement of Theorem 4.1 we have conditions which are similar to those in the case $n=2$, but depending on the reaction rate constants corresponding to the layers $i_{0}$ and $i_{0}+1$ and total conservation constants. Again, the two sets of inequalities in the statement of Theorem 4.1 are clearly compatible.

For $n \geq 3$, there is not only an increase in the number of variables but also in the number of conservation laws. The idea of the proof of Theorem 4.1 is to extend the simplices that appear in the proof of Theorem 3.1 to simplices in the higher dimensional space, showing that in fact the conditions of the new simplices to be positively decorated are basically the same.

The other case is when the layers which share a phosphatase are not consecutive. Assume $i_{1}<i_{2}$ are two non-consecutive layers sharing the same phosphatase. Assume also that there are no two other consecutives layers with a common phosphatase between them (otherwise, we would be in the hyphothesis of the previous case). That is, there exists $i_{1}, i_{2}$, with $1 \leq i_{1}<i_{1}+1<i_{2} \leq n$, such that $P_{j\left(i_{1}\right)}=P_{j\left(i_{2}\right)}=F$, and $P_{j(i)}$ for $i=i_{1}+1, \ldots, i_{2}-1$ are all distinct and different from $F$. We impose no restrictions on the phosphatases of the remaining layers layers $1, \ldots, i_{1}-1, i_{2}+1, \ldots, n$. 
Consider the following rational functions $\beta_{1, i_{1}, i_{2}}, \beta_{2, i_{1}, i_{2}}, \beta_{3, i_{1}, i_{2}}$ and $\beta_{4, i_{1}, i_{2}}$ depending on the catalytic reaction rate constant and total concentration constants:

$$
\begin{aligned}
& \beta_{1, i_{1}, i_{2}}=\frac{S_{i_{1}-1, t o t}}{S_{i_{1}, t o t}}-\frac{A_{i_{1}}}{A_{i_{1}+1}}, \\
& \beta_{2, i_{1}, i_{2}}=\left(A_{i_{1}}+1\right)-\frac{S_{i_{1}, t o t}}{F_{t o t}}, \\
& \beta_{3, i_{1}, i_{2}}=\frac{S_{i_{2}-1, t o t}}{F_{t o t}}-\left(A_{i_{1}}+1\right) \frac{S_{i_{2}, t o t}}{F_{t o t}}, \\
& \beta_{4, i_{1}, i_{2}}=\frac{S_{i_{1}, t o t}}{F_{t o t}}-\left(\frac{A_{i_{1}}+1}{A_{i_{2}}+1}\right)\left(A_{i_{2}}+1-\frac{S_{i_{2}, t o t}}{F_{t o t}}\right),
\end{aligned}
$$

where $F_{t o t}=P_{j\left(i_{1}\right), t o t}=P_{j\left(i_{2}\right), t o t}$.

We then have:

Theorem 4.3. Suppose $n \geq 3$, and suppose there exists layers $i_{1}, i_{2}$, with $1 \leq i_{1}<$ $i_{1}+1<i_{2} \leq n$, such that $P_{j\left(i_{1}\right)}=P_{j\left(i_{2}\right)}=F, P_{j(i)}$ for $i=i_{1}+1, \ldots, i_{2}-1$ are all distinct and different from $F$, and with no restriction in the phosphatases of layers $1, \ldots, i_{1}-1, i_{2}+1, \ldots, n$. Assume the reaction rate constants and the total concentration constants verify

$$
\beta_{1, i_{1}, i_{2}}, \beta_{2, i_{1}, i_{2}}, \beta_{3, i_{1}, i_{2}}, \beta_{4, i_{1}, i_{2}}>0 .
$$

Then, there exists a rescaling in the constants $k_{\text {on }_{i}}, i=1, \ldots, n$ and $\ell_{o n_{i}}, i=1, \ldots, n$, such that the system has at least two positive steady states.

Again, we will give an explicit rescaling in the proof.

Remark 4.4. The inequalities in the statement of Theorem 4.3 are compatible. They have a similar flavour, but they are different from the conditions defining the regions of multistationarity in Theorems 3.1 and 4.1.

\subsection{The proof of Theorem 4.1.}

Proof of Theorem 4.1. Without loss of generality we suppose that the phosphatase in the layers $i_{0}$ and $i_{0}+1$ is the phosphatase $P_{1}$, that we call $F$. We showed in (4.1) that we can parametrize the steady states in terms of the concentrations of $s_{i}^{1}$, for $i=0, \ldots, n, f$ (we use $f$ instead of $p_{1}$ ) and $p_{i}$, for $i=2, \ldots, r$. To avoid unnecessary notation, in this proof we call $s_{i}=s_{i}^{1}$ for all $i=0, \ldots, n$.

Consider the following set of monomials:

$$
\mathcal{M}=\left\{s_{i_{0}-1}, f, s_{i_{0}}, s_{i_{0}+1}, s_{i_{0}} f, s_{i_{0}+1} f, s_{i_{0}} f\left(s_{i_{0}-1}\right)^{-1}, s_{i_{0}+1} f\left(s_{i_{0}}\right)^{-1}, 1\right\} .
$$

These monomials appear in the parametrization of the concentration at steady state of the species in layers $i_{0}$ and $i_{0}+1$. Now, consider the set

$$
\mathcal{M}^{\prime}=\mathcal{M} \cup\left\{s_{0}, s_{1}, \ldots, s_{i_{0}-2}, s_{i_{0}+2}, \ldots, s_{n}, p_{2}, \ldots, p_{r}\right\} .
$$

And consider also the set of all the monomials that appear in the parametrization:

$$
\begin{aligned}
\mathcal{M}^{\prime \prime}=\mathcal{M}^{\prime} \cup & \left\{s_{1} p_{j(1)}, \ldots, s_{i_{0}-1} p_{j\left(i_{0}-1\right)}, s_{i_{0}+2} p_{j\left(i_{0}+2\right)}, \ldots, s_{n} p_{j(n)}, s_{1} p_{j(1)}\left(s_{0}\right)^{-1}, \ldots\right. \\
& \left.\ldots, s_{i_{0}-1} p_{j\left(i_{0}-1\right)}\left(s_{i_{0}-2}\right)^{-1}, s_{i_{0}+2} p_{j\left(i_{0}+2\right)}\left(s_{i_{0}+1}\right)^{-1}, \ldots, s_{n} p_{j(n)}\left(s_{n-1}\right)^{-1}\right\} .
\end{aligned}
$$

We have $n+r+1$ variables: $s_{0}, s_{1}, \ldots, s_{n}, f, p_{2}, \ldots, p_{r}$. Consider the variables with this last order. Let $\mathcal{A}, \mathcal{A}^{\prime}, \mathcal{A}^{\prime \prime} \subset \mathbb{R}^{n+r+1}$ be the subsets corresponding to the supports of the sets $\mathcal{M}, \mathcal{M}^{\prime}, \mathcal{M}^{\prime \prime}$ respectively, that is, the exponents of the monomials in each set.

We consider an order in $\mathcal{A}^{\prime \prime}$ given by the order in which we construct $\mathcal{M}^{\prime \prime}$ : first the exponents corresponding to monomials in $\mathcal{M}$ (in that order), then the exponents corresponding to monomials that we add to obtain $\mathcal{M}^{\prime}$ (in that order), and then the rest of the exponents, in the same order as enumerated above. We have $3 n+r+2$ monomials.

As in the case $n=2$, we replace the monomial parametrization into the conservation laws (4.1) and we write this system in a matricial form. We call $C \in \mathbb{R}^{(n+r+1) \times(3 n+r+2)}$ the matrix of coefficients of the resulting polynomial system. 
We want to find two simplices with vertices in $\mathcal{A}^{\prime \prime}$ which share a facet. Inspired by the 4 -simplices that we chooses for the case $n=2$, we take the following $(n+r+1)$-simplices:

$$
\begin{aligned}
& \Delta_{1}=\left\{e_{i_{0}}, e_{i_{0}+1}+e_{n+2}, e_{i_{0}+2}+e_{n+2}, e_{i_{0}+2}, 0\right\} \cup\left(\mathcal{A}^{\prime} \backslash \mathcal{A}\right), \\
& \Delta_{2}=\left\{e_{i_{0}}, e_{i_{0}+1}+e_{n+2}, e_{i_{0}+2}+e_{n+2}, e_{i_{0}+2}+e_{n+2}-e_{i_{0}+1}, 0\right\} \cup\left(\mathcal{A}^{\prime} \backslash \mathcal{A}\right) .
\end{aligned}
$$

where $e_{i}$ denotes the $i$-th canonical vector of $\mathbb{R}^{n+r+1}$. Note that the points $e_{i_{0}}, e_{i_{0}+2}$, $e_{i_{0}+1}+e_{n+2}, e_{i_{0}+2}+e_{n+2}, 0$ correspond to the monomials $s_{i_{0}-1}, s_{i_{0}+1}, s_{i_{0}} f, s_{i_{0}+1} f, 1$, and the points $e_{i_{0}}, e_{i_{0}+1}+e_{n+2}, e_{i_{0}+2}+e_{n+2}, e_{i_{0}+2}+e_{n+2}-e_{i_{0}+1}, 0$, correspond to the monomials $s_{i_{0}-1}, s_{i_{0}} f, s_{i_{0}+1} f, s_{i_{0}+1} f\left(s_{i_{0}}\right)^{-1}, 1$ which are in correspondence with the points of the simplices in the proof of Theorem 3.1.

We consider first the equations corresponding to the conservation laws with total conservation constants $S_{i_{0}-1, t o t}, F_{t o t}, S_{i_{0}, t o t}, S_{i_{0}+1, t o t}$ and then the equations corresponding to the conservation constants $S_{0, t o t}, \ldots, S_{i_{0}-2, t o t}, S_{i_{0}+2, t o t}, \ldots, S_{n, t o t}, P_{2, t o t}, \ldots, P_{n, t o t}$. The submatrices of $C$ restricted to the columns corresponding to the simplex $\Delta_{j}$, for $j=1,2$, are equal to:

$$
C_{\Delta_{j}}=\left(\begin{array}{cccc|c} 
& \multicolumn{3}{c|}{C_{j}} & 0 \\
\hline 0 & \ldots & 0 & -S_{0, t o t} & \\
\vdots & \ddots & \vdots & \vdots & \\
0 & \ldots & 0 & -S_{i_{0}-2, t o t} & \\
0 & \ldots & 0 & -S_{i_{0}+2, t o t} & \\
\vdots & \ddots & \vdots & \vdots & \operatorname{Id}_{n+r-3} \\
0 & \ldots & 0 & -S_{n, t o t} & \\
0 & \ldots & 0 & -P_{2, t o t} & \\
\vdots & \ddots & \vdots & \vdots & \\
0 & \ldots & 0 & -P_{r, t o t} &
\end{array}\right)
$$

where $C_{1}$ is the submatrix corresponding to columns of the exponents $\left\{e_{i_{0}}, e_{i_{0}+1}+\right.$ $\left.e_{n+2}, e_{i_{0}+2}+e_{n+2}, e_{i_{0}+2}, 0\right\}$ and $C_{2}$ is the submatrix corresponding to the columns of the exponents $\left\{e_{i_{0}}, e_{i_{0}+1}+e_{n+2}, e_{i_{0}+2}+e_{n+2}, e_{i_{0}+2}+e_{n+2}-e_{i_{0}+1}, 0\right\}$, that is:

$$
C_{j}=\left(\begin{array}{ccccc}
1 & K_{i_{0}} G_{i_{0}} & 0 & 0 & -S_{i_{0}-1, t o t} \\
0 & L_{i_{0}} & L_{i_{0}+1} & 0 & -F_{t o t} \\
0 & K_{i_{0}} G_{i_{0}}+L_{i_{0}} & K_{i_{0}+1} G_{i_{0}+1} & 0 & -S_{i_{0}, t o t} \\
0 & 0 & K_{i_{0}+1} G_{i_{0}+1}+L_{i_{0}+1} & \left(C_{j}\right)_{44} & -S_{i_{0}+1, t o t}
\end{array}\right),
$$

where $\left(C_{1}\right)_{44}=1$ and $\left(C_{2}\right)_{44}=G_{i_{0}+1}$.

Note that the matrix $C_{\Delta_{j}}$ is positively spanning if and only if $C_{j}$ is positively spanning, for $j=1,2$. Moreover, $C_{1}$ and $C_{2}$ are positively spanning if and only if the condition of the statement holds, that is, $A_{i_{0}}+1>A_{i_{0}+1}$ and $\alpha_{1, i_{0}}, \alpha_{2, i_{0}}, \alpha_{3, i_{0}}, \alpha_{4, i_{0}}>0$; or $A_{i_{0}}+1<A_{i_{0}+1}$ and $\alpha_{1, i_{0}}, \alpha_{2, i_{0}}, \alpha_{3, i_{0}}, \alpha_{4, i_{0}}<0$.

Given $h \in \mathcal{C}_{\Delta_{1}, \Delta_{2}}$, by Theorem 2.7 there exists $t_{0} \in \mathbb{R}_{>0}$ such that for all $0<t<t_{0}$, the number of positive (nondegenerate) solutions of the scaled system, that is, the system with the same support $\mathcal{A}^{\prime \prime}$ and matrix of coefficients $C_{t}$ with $\left(C_{t}\right)_{i j}=t^{h\left(\alpha_{j}\right)} c_{i j}$, with $\alpha_{j} \in \mathcal{A}^{\prime \prime}$ and $C=\left(c_{i j}\right)$, is at least two. This system has the following form. Call $x=\left(s_{0}, s_{1}, \ldots, s_{n}, f, p_{2}, \ldots, p_{r}\right)$ and note that each coefficient $c_{i j}$ is a rational function of the vector of reaction rate constants that we call $\kappa=\left(k_{o n_{1}}, \ell_{o n_{1}}, \ldots,\right)$. To emphasize this, we write $c_{i j}=c_{i j}(\kappa)$. Moreover, setting $\gamma_{j}=t^{h\left(\alpha_{j}\right)}$ for any $j$, we have a Laurent polynomial system of $n+r+1$ equations in $n+r+1$ variables:

$$
\sum_{j} c_{i j}(\kappa) \gamma_{j} x^{\alpha_{j}}=0, i=1, \ldots, n+r+1 .
$$

Now, the reaction network we are considering satisfies the hypotheses of Theorem 5.4 of [4]. Then, there exists a vector of reaction rate constants $\bar{\kappa}$ such that the number of positive solutions of system (4.4) coincides with the number of positive solutions of the 
following system:

$$
\sum_{j} c_{i j}(\bar{\kappa}) x^{\alpha_{j}}=0, i=1, \ldots, n+r+1
$$

We now describe the associated vector $\bar{\kappa}$ in an explicit form. We first describe the cone $\mathcal{C}_{\Delta_{1}, \Delta_{2}}$ defined in (2.4). We denote by $h_{j}$ the height corresponding to $\alpha_{j} \in \mathcal{A}^{\prime \prime}$, for $j=1, \ldots, 3 n+r+2$ (in the order corresponding to the construction of $\mathcal{M}^{\prime \prime}$ that we described before). Let $\varphi_{1}$ and $\varphi_{2}$ be the affine linear functions which agree with $h$ on the simplices $\Delta_{1}$ and $\Delta_{2}$ respectively. We can take the heights of the points of $\Delta_{1}$ as zero, that is, $h_{1}=h_{4}=h_{5}=h_{6}=h_{9}=0$ and $h_{j}=0$ for $j=10, \ldots, n+r+1$, and $h_{8}>0$ (the height of the remaining point of $\Delta_{2}$ which is not in $\left.\Delta_{1}\right)$. Then, $\varphi_{1}\left(x_{1}, \ldots, x_{n+r+1}\right)=0$ and $\varphi_{2}\left(x_{1}, \ldots, x_{n+r+1}\right)=-h_{8} x_{i_{0}+1}-h_{8} x_{i_{0}+2}+h_{8} x_{n+2}$. Moreover, $h$ satisfies $0=\varphi_{1}(\alpha)<$ $h(\alpha)$, for all $\alpha \notin \Delta_{1}$ and $\varphi_{2}(\alpha)<h(\alpha)$, for all $\alpha \notin \Delta_{1}, \Delta_{2}$. Then, we have:

$$
\begin{aligned}
& h_{8}<h_{2}, \quad 0<h_{3}, \quad 0<h_{7}, \\
& h_{j}>0, \text { for } j=n+r+7, \ldots, 3 n+r+2, \\
& h_{8}<h_{n+r+6+j}, \text { for } j \in \Lambda_{1}, \text { with } j=1, \ldots, i_{0}-1, \\
& h_{8}<h_{n+r+4+j}, \text { for } j \in \Lambda_{1}, \text { with } j=i_{0}+2, \ldots, n, \\
& h_{8}<h_{2 n+r+4+j} \text { for } j \in \Lambda_{1}, \text { with } j=1, \ldots, i_{0}-1, \\
& 2 h_{8}<h_{2 n+r+4+i}, \text { if } i_{0}+2 \in \Lambda_{1}, \text { and } h_{8}<h_{2 n+r+2+j} \text { for } j \in \Lambda_{1}, \text { with } j=i_{0}+3, \ldots, n .
\end{aligned}
$$

where $h_{2}, h_{3}, h_{7}$ and $h_{j}, j=n+r+7, \ldots, 3 n+r+2$, are generic.

If we change the variables $\bar{f}=t^{h_{2}} f, \bar{s}_{i_{0}}=t^{h_{3}} s_{i_{0}}$, we consider the constants:

$$
\begin{aligned}
\overline{K_{i_{0}}} & =t^{-h_{7}} K_{i_{0}}, \quad \overline{K_{i_{0}+1}}=t^{-h_{3}-h_{8}} K_{i_{0}+1}, \quad \overline{L_{i_{0}}}=t^{-h_{2}-h_{3}} L_{i_{0}}, \quad \overline{L_{i_{0}+1}}=t^{-h_{2}} L_{i_{0}+1}, \\
\overline{K_{i}} & =t^{h_{n+r+6+i}-h_{2 n+r+4+i}} K_{i}, i=1, \ldots, i_{0}-1, \\
\text { (4.6) } \overline{K_{i}} & =t^{h_{n+r+4+i}-h_{2 n+r+2+i}} K_{i}, i=i_{0}+2, \ldots, n, \\
\overline{L_{i}} & =t^{h_{n+r+6+i}-h_{2}} L_{i}, \text { if } i \in \Lambda_{1}, \overline{L_{i}}=t^{h_{n+r+6+i}} L_{i}, \text { if } i \notin \Lambda_{1}, \text { for } i=1, \ldots, i_{0}-1, \\
\overline{L_{i}} & =t^{h_{n+r+4+i}-h_{2}} L_{i}, \text { if } i \in \Lambda_{1}, \overline{L_{i}}=t^{h_{n+r+4+i}} L_{i}, \text { if } i \notin \Lambda_{1}, \text { for } i=i_{0}+2, \ldots, n,
\end{aligned}
$$

and we keep fixed the values of the constants $k_{\text {cat }_{\mathrm{i}_{0}}}, k_{\text {cat }_{0}+1}, \ell_{\text {cat }_{\mathrm{i}_{0}}}$ and $\ell_{\text {cat }_{\mathrm{i}_{0}+1}}$ and the total conservation constants $S_{i_{0}-1, t o t}, F_{t o t}, S_{i_{0}, t o t}$ and $S_{i_{0}+1, t o t}$, then the dynamical system associated with the network with these constants is system (4.5) with coefficients depending on the scaled reaction constants. Therefore, the cascade we are considering has at least two positive steady states for these constants.

To get the scalings in (4.6) it can be checked that it is enough to rescale the original constants as follows:

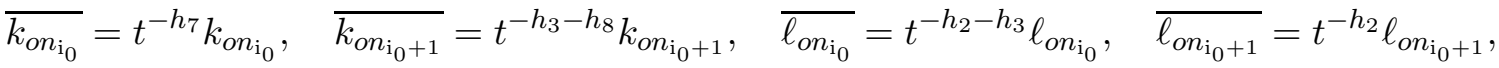

$$
\begin{aligned}
& \overline{k_{o n_{\mathrm{i}}}}=t^{h_{n+r+6+i}-h_{2 n+r+4+i}} k_{o n_{\mathrm{i}}}, i=1, \ldots, i_{0}-1 \text {, } \\
& \overline{k_{o n_{\mathrm{i}}}}=t^{h_{n+r+4+i}-h_{2 n+r+2+i}} k_{o n_{\mathrm{i}}}, i=i_{0}+2, \ldots, n \text {, } \\
& \overline{\ell_{o n_{\mathrm{i}}}}=t^{h_{n+r+6+i}-h_{2}} \ell_{o n_{\mathrm{i}}} \text {, if } i \in \Lambda_{1}, \overline{\ell_{o n_{\mathrm{i}}}}=t^{h_{n+r+6+i}} \ell_{o n_{\mathrm{i}}} \text {, if } i \notin \Lambda_{1} \text {, for } i=1, \ldots, i_{0}-1 \text {, } \\
& \overline{\ell_{o n_{\mathrm{i}}}}=t^{h_{n+r+4+i}-h_{2}} \ell_{o n_{\mathrm{i}}} \text {, if } i \in \Lambda_{1}, \overline{\ell_{o n_{\mathrm{i}}}}=t^{h_{n+r+4+i}} \ell_{o n_{\mathrm{i}}} \text {, if } i \notin \Lambda_{1} \text {, for } i=i_{0}+2, \ldots, n \text {. }
\end{aligned}
$$

4.4. The proof of Theorem 4.3. For simplicity and to fix ideas, we only present a proof in the case when the first and the last layer have the same phosphatase (that is, $\left.i_{1}=1, i_{2}=n\right)$, and the other layers have all different phosphatases. We also present an explicit rescaling for this case. The general case is similar, but with a heavier notation.

Proof of Theorem 4.3. We call $f$ the concentration of the phosphatase $F$, the phosphatase that appear in the layers 1 and $n$, and we call $f_{i}=p_{j(i)}$ for $i=2, \ldots, n-1$. By assumption the variables $f_{i}$ are all distinct and different from $f$. We showed in (4.1) that we can 
parametrize the steady states in terms of the concentrations of $s_{i}^{1}$, for $i=0, \ldots, n, f$ and $f_{i}$, for $i=2, \ldots, n-1$. To avoid unnecessary notation, in this proof we call again $s_{i}=s_{i}^{1}$ for all $i=0, \ldots, n$.

We have $2 n$ variables, and we consider these $2 n$ variables with the following order: $s_{0}, s_{1}, \ldots, s_{n}, f, f_{2}, \ldots, f_{n-1}$. In the monomial parametrization there are $4 n+1$ different monomials, and we consider these monomials with this order: $s_{0}, s_{1}, \ldots, s_{n}, f$, $f_{2}, \ldots, f_{n-1}, s_{1} f, s_{2} f_{2}, \ldots, s_{n-1} f_{n-1}, s_{n} f, s_{1} f\left(s_{0}\right)^{-1}, s_{2} f_{2}\left(s_{1}\right)^{-1}, \ldots, s_{n-1} f_{n-1}\left(s_{n-2}\right)^{-1}$, $s_{n} f\left(s_{n-1}\right)^{-1}, 1$.

As in the previous cases, we replace the monomial parametrization into the conservation laws and we write this system in matricial form. Let $C \in \mathbb{R}^{(2 n) \times(4 n+1)}$ be the matrix of coefficients of this polynomial system. We call $\mathcal{A}$ the support of the system.

We want to find two simplices with vertices in $\mathcal{A}$ which share a facet. We denote $\mathcal{B} \subset \mathcal{A}$ the set of the exponents corresponding to the monomials: $s_{2} f_{2}\left(s_{1}\right)^{-1}, \ldots, s_{n-2} f_{n-2}\left(s_{n-3}\right)^{-1}$, $f_{2}, \ldots, f_{n-1}$. We consider the two following simplices: $\Delta_{1}$ given by the exponents corresponding to the monomials $s_{0}, s_{1} f, s_{n} f, s_{n-1} f_{n-1}\left(s_{n-2}\right)^{-1}, s_{n}, 1$, and the points in $\mathcal{B}$, and $\Delta_{2}$ given by the exponents corresponding to the monomials $s_{0}, s_{1} f, s_{n} f$, $s_{n-1} f_{n-1}\left(s_{n-2}\right)^{-1}, s_{n} f\left(s_{n-1}\right)^{-1}, 1$ and the points in $\mathcal{B}$. That is:

$$
\begin{aligned}
& \Delta_{1}=\left\{e_{1}, e_{2}+e_{n+2}, e_{n+1}+e_{n+2}, e_{n}+e_{2 n}-e_{n-1}, e_{n+1}, 0\right\} \cup \mathcal{B}, \\
& \Delta_{2}=\left\{e_{1}, e_{2}+e_{n+2}, e_{n+1}+e_{n+2}, e_{n}+e_{2 n}-e_{n-1}, e_{n+1}+e_{n+2}-e_{n}, 0\right\} \cup \mathcal{B},
\end{aligned}
$$

where $e_{i}$ denotes the $i$-th canonical vector of $\mathbb{R}^{2 n}$.

If we consider first the equations corresponding to the conservation laws with total conservation constants $S_{0, t o t}, F_{t o t}, S_{1, t o t}, S_{n-1, t o t}, S_{n, t o t}$ and then the equations corresponding to the conservation constants $S_{2, t o t}, \ldots, S_{n-2, t o t}, F_{2, t o t}, \ldots, F_{n-1, t o t}$, the submatrices of $C$ restricted to the columns corresponding to the simplices $\Delta_{j}$ for $j=1,2$ are as follows. We change the order of the columns following the order of the monomials in each simplex; the property of being positively spanning remains invariant:

$$
C_{\Delta_{j}}=\left(\begin{array}{cccc|c|c}
\multicolumn{3}{c|}{C_{j}} & 0 & 0 \\
\hline 0 & \ldots & 0 & -S_{2, t o t} & & \\
\vdots & \ddots & \vdots & \vdots & G & 0 \\
0 & \ldots & 0 & -S_{n-2, t o t} & & \\
\hline 0 & \ldots & 0 & -F_{2, t o t} & & \\
\vdots & \ddots & \vdots & \vdots & 0 & \operatorname{Id}_{n-2} \\
0 & \ldots & 0 & -F_{n-1, t o t} & &
\end{array}\right),
$$

where $G \in \mathbb{R}^{(n-3) \times(n-3)}$ is the diagonal matrix with $G_{i i}=G_{i+1}$, for $i=1, \ldots, n-3, C_{1}$ is the submatrix corresponding to columns of the exponents $\left\{e_{1}, e_{2}+e_{n+2}, e_{n+1}+e_{n+2}, e_{n}+\right.$ $\left.e_{2 n}-e_{n-1}, e_{n+1}, 0\right\}$ and $C_{2}$ is the submatrix corresponding to the columns of the exponents $\left\{e_{1}, e_{2}+e_{n+2}, e_{n+1}+e_{n+2}, e_{n}+e_{2 n}-e_{n-1}, e_{n+1}+e_{n+2}-e_{n}, 0\right\}:$

$$
C_{j}=\left(\begin{array}{cccccc}
1 & K_{1} G_{1} & 0 & 0 & 0 & -S_{0, t o t} \\
0 & L_{1} & L_{n} & 0 & 0 & -F_{t o t} \\
0 & K_{1} G_{1}+L_{1} & 0 & 0 & 0 & -S_{1, t o t} \\
0 & 0 & K_{n} G_{n} & G_{n-1} & 0 & -S_{n-1, t o t} \\
0 & 0 & K_{n} G_{n}+L_{n} & 0 & \left(C_{j}\right)_{55} & -S_{n, t o t}
\end{array}\right),
$$

with $\left(C_{1}\right)_{55}=1$ and $\left(C_{2}\right)_{55}=G_{n}$.

We observe that the matrix $C_{\Delta_{j}}$ if positively spanning if and only if $C_{j}$ is positively spanning, for $i=1,2$. It is straightforward to check that the conditions under which $C_{1}$ and $C_{2}$ are positively spanning are equivalent to the conditions of the statement: $\beta_{1,1, n}$, $\beta_{2,1, n}, \beta_{3,1, n}, \beta_{4,1, n}>0$.

Given $h \in \mathcal{C}_{\Delta_{1}, \Delta_{2}}$, by Theorem 2.7 there exists $t_{0} \in \mathbb{R}_{>0}$ such that for all $0<t<t_{0}$, the number of positive (nondegenerate) solutions of the scaled system, i.e. the system with 
support $\mathcal{A}$ and matrix of coefficients $C_{t}$, with $\left(C_{t}\right)_{i j}=t^{h\left(\alpha_{j}\right)} c_{i j}$, (with $\left.\alpha_{j} \in \mathcal{A}, C=\left(c_{i j}\right)\right)$ is at least two.

We now argue as in the proof of Theorem 4.1. We can write our system in the form (4.4), and since any cascade of Goldbeter-Koshland loops satisfies the hypotheses of Theorem 5.4 of [4], we again deduce the existence of a vector of rate constants $\bar{\kappa}$ such that the number of positive solutions of this system coincides with the number of positive solutions of the corresponding system of the form (4.5). In what follows, we also explicitly describe the rescaling of the parameters.

We denote by $h_{j}$ the height corresponding to $\alpha_{j} \in \mathcal{A}$, for $j=1, \ldots, 4 n+1$, with the order of the monomials as we described before. Let $\varphi_{1}$ and $\varphi_{2}$ be the affine linear functions which agree with $h$ on the simplices $\Delta_{1}$ and $\Delta_{2}$ respectively.

We can take zero heights at the points of $\Delta_{1}$, that is, $h_{1}=h_{2 n+1}=h_{3 n}=h_{4 n-1}=$ $h_{n+1}=h_{4 n+1}=0, h_{j}=0$ for $j=n+3, \ldots, 2 n, h_{j}=0$, for $j=3 n+2, \ldots, 4 n-2=0$ if $n>$ 3 (note that if $n=3, h_{3 n+2}=h_{4 n-1}$, already defined) and $h_{4 n}>0$ (the height of the other point of $\left.\Delta_{2}\right)$. Then, $\varphi_{1}\left(x_{1}, \ldots, x_{2 n}\right)=0$ and $\varphi_{2}\left(x_{1}, \ldots, x_{2 n}\right)=-h_{4 n} \sum_{i=2}^{n} x_{i}+h_{4 n} x_{n+2}$.

As $h \in \mathcal{C}_{\Delta_{1}, \Delta_{2}}$, we have that $h$ satisfies $0=\varphi_{1}(\alpha)<h(\alpha)$, for all $\alpha \notin \Delta_{1}$ and $\varphi_{2}(\alpha)<$ $h(\alpha)$, for all $\alpha \notin \Delta_{1}, \Delta_{2}$. Then, we have these conditions:

$$
h_{4 n}<h_{n+2}, \quad h_{j}>0, \text { for } j=2, \ldots, n, 2 n+2, \ldots, 3 n-1,3 n+1,
$$

where $h_{j}$ for $j=2, \ldots, n, n+2,2 n+2, \ldots, 3 n-1,3 n+1$ are generic.

If we change the variables $\bar{s}_{i}=t^{h_{i+1}} s_{i}$, for $i=1, \ldots, n-1$ and $\bar{f}=t^{h_{n+2}} f$, we consider the constants:

$$
\begin{aligned}
\overline{K_{1}} & =t^{-h_{3 n+1}} K_{1}, & \overline{L_{1}} & =t^{-h_{2}-h_{n+2}} L_{1}, \\
\overline{K_{i}} & =t^{h_{2 n+i}-h_{i}} K_{i}, & \overline{L_{i}} & =t^{h_{2 n+i}-h_{i+1}} L_{i},
\end{aligned} \quad \text { for } i=2, \ldots, n-1,
$$

without altering the values of the constants $k_{\mathrm{cat}_{1}}, k_{\mathrm{cat}_{\mathrm{n}}}, \ell_{\mathrm{cat}_{1}}, \ell_{\mathrm{cat}_{\mathrm{n}}}$ and the total conservation values, then the dynamical system associated with the network with these constants is the scaled system. Therefore, the network has at least two positive steady states for this choice of constants.

It is straightforward to check that to get the scalings in (4.8) it is enough to rescale the original constants as follows:

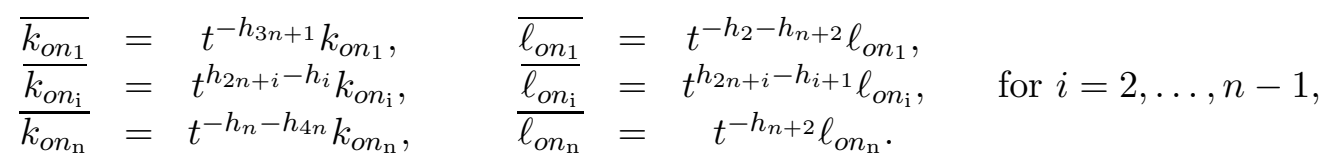

\section{Acknowledgment}

The authors are grateful to the Kurt and Alice Wallenberg Foundation and to the Institut Mittag-Leffler, Sweden, for their support to work on this project. We are also grateful to the Mathematics Department of the Royal Institute of Technology, Sweden, for the wonderful hospitality we enjoyed, and to the French Program PREFALC and the University of Buenos Aires, which made possible the visit of F. Bihan.

\section{REFERENCES}

[1] M. Banaji, C. Pantea. The inheritance of nondegenerate multistationarity in chemical reaction networks. SIAM J. Appl. Math. 78(2) (2018), 1105-1130.

[2] S. Basu, R. Pollack, M.-F. Roy. Algorithms in Real Algebraic Geometry, volume 10 of Algorithms and Computation in Mathematics. Springer-Verlag Berlin Heidelberg, 2nd. ed., 2016.

[3] A. Barabanschikov, J. Gunawardena. Monostationarity and multistationarity in tree networks of Goldbeter-Koshland loops. Preprint.

[4] F. Bihan, A. Dickenstein, M. Giaroli. Lower bounds for positive roots and regions of multistationarity in chemical reaction networks. Preprint, available at: arXiv:1807.05157 (2018).

[5] F. Bihan, F. Santos, P-J. Spaenlehauer. A polyhedral method for sparse systems with many positive solutions. Preprint, available at: arXiv:1804.05683 (2018). 
[6] S. Catozzi, J. P. Di-Bella, A. C. Ventura, J. A. Sepulchre. Signaling cascades transmit information downstream and upstream but unlikely simultaneously. BMC Systems Biology, 10(1), 84, (2016).

[7] C. Conradi, E. Feliu, M. Mincheva, C. Wiuf. Identifying parameter regions for multistationarity. PLoS Comput Biol 13(10) (2017): e1005751.

[8] C. Conradi, D. Flockerzi, J. Raisch. Multistationarity in the activation of a MAPK: Parametrizing the relevant region in parameter space. Mathematical biosciences 211 (1) (2012), 105-131

[9] C. Conradi, M. Mincheva. Catalytic constants enable the emergence of bistability in dual phosphorylation. J. R. Soc. Interface (2014), rsif20110664.

[10] G. Craciun, M. Feinberg. Multiple equilibria in complex chemical reaction networks: I. The injectivity property. SIAM J. Appl. Math. 65 (2005), 1526-1546.

[11] G. Craciun, M. Feinberg. Multiple equilibria in complex chemical reaction networks: II. The Species-Reactions Graph. SIAM J. Appl. Math. 66(4) (2006), 1321-1338.

[12] J. de Loera, J.A. Rambau, F. Santos. Triangulations: Structures for Algorithms and Applications, vol. 25. Springer-Verlag, 2010.

[13] W. Decker; G.-M. Greuel ; G. Pfister; H. Schönemann: Singular 4-1-1 - A computer algebra system for polynomial computations. http://www.singular.uni-kl.de (2018).

[14] E. Feliu, M. Knudsen, L. N. Andersen, C. Wiuf. (2012). An algebraic approach to signaling cascades with $n$ layers. Bulletin of mathematical biology, 74(1), (2012) 45-72.

[15] E. Feliu, C. Wiuf. Enzyme-sharing as a cause of multi-stationarity in signaling systems J. R. Soc. Interface 9 (2012), 1224-1232.

[16] E. Feliu, C. Wiuf Simplifying biochemical models with intermediate species. J. R. Soc. Interface (2013) 10: 20130484.

[17] D. Flockerzi, C. Conradi. Subnetwork analysis for multistationarity in mass action kinetics. J. of Physics: Conference Series 138 (2008), 012006.

[18] K. Gatermann, M. Wolfrum. Bernstein's second theorem and Viro's method for sparse polynomial systems in chemistry. Advances in Applied Mathematics, 34(2), (2005), 252-294.

[19] A. Goldbeter, D. E. Koshland. An amplified sensitivity arising from covalent modification in biological systems. Proceedings of the National Academy of Sciences, 78(11), (1981) 6840-6844.

[20] Y. Goyal, et al. Divergent effects of intrinsically active MEK variants on developmental Ras signaling. Nature genetics 49.3 (2017), 465.

[21] K. Holstein, D. Flockerzi, C. Conradi. Multistationarity in Sequential Distributed Multisite Phosphorylation Networks. Bull. Math. Biol. 75 (11) (2013), 2028-2058.

[22] B. Joshi, A. Shiu. Atoms of multistationarity in chemical reaction networks. J. Math. Chem. 51(1) (2013), 153-178.

[23] V. B. Kothamanchu, E. Feliu, L. Cardelli, O. S. Soyer. Unlimited multistability and Boolean logic in microbial signaling. J. R. Soc. Interface (2015), 1220150234.

[24] L. Li, et al. The Ras/Raf/MEK/ERK signaling pathway and its role in the occurrence and development of HCC. Oncology letters, vol. 12, no 5, (2016) 3045-3050.

[25] S. Mueller, E. Feliu, G. Regensburger, C. Conradi, A. Shiu, A. Dickenstein. Sign conditions for injectivity of generalized polynomial maps with applications to chemical reaction networks and real algebraic geometry. FoCM Journal 16 (1) (2016), 69-97.

[26] M. Pérez Millán, A. Dickenstein. The structure of MESSI biochemical networks. SIAM J. Appl. Dyn. Sys. 17(2) (2018), 1650-1682.

[27] M. Pérez Millán, A. Turjanski. MAPK's networks and their capability for multistationarity due to toric steady states. Math. Biosciences 262 (2015), 125-137.

[28] L. Wang, E. Sontag. On the number of steady states in a multiple futile cycle. J. Math. Biol. $57(1)(2008), 29-52$.

\section{Appendix A. General statements Behind our Results about Cascades}

In the proof of Theorem 4.1 we extrapolated the multistationarity behaviour and the description of a region of multistationarity of a subnetwork (described in 3.1) to the whole network, even if it has more linearly independent conservation relations. For this, we developed some ideas that we now abstract in Theorem A.3 and that can be used in the study of other cascade mechanisms. As we remarked at the end of the Introduction, they can be applied to describe a multistationarity region for the Ras cascade in Figure 1 (see [27] for details about different models), extrapolating our results about a single layer with two sequential phosporilations proved in Theorem 4.1 in [4]. We assume the reader is familiar with the content of Section 2 in our companion paper [4]. 
We start with a couple of lemmas. Given a lattice configuration $\mathcal{A}$, we will denote by $\operatorname{Aff}(\mathcal{A})$ the affine span of $\mathcal{A}$ consisting of of all points $\sum_{a \in \mathcal{A}} \lambda_{a} \cdot a$ with $\lambda_{a} \in \mathbb{Z}$ for all $a \in \mathcal{A}$ and $\sum_{a \in \mathcal{A}} \lambda_{a}=0$.

Lemma A.1. Let $\mathcal{A} \subset \mathcal{A}^{\prime} \subset \mathbb{Z}^{d}$ be finite point configurations, with $\operatorname{Aff}(\mathcal{A})=\operatorname{Aff}\left(\mathcal{A}^{\prime}\right)=\mathbb{Z}^{d}$. Suppose that $\tau$ is a regular subdivision of $\mathcal{A}$. Then, there exists a regular subdivision $\tau^{\prime}$ of $\mathcal{A}^{\prime}$ such that $\tau \subset \tau^{\prime}$. Moreover, we can choose a lifting function $h^{\prime}$ inducing $\tau^{\prime}$ such that $h:=\left.h^{\prime}\right|_{\mathcal{A}}$ induces $\tau$.

Proof. Let $h_{\tau}: \mathcal{A} \rightarrow \mathbb{R}$ be any lifting function inducing the subdivision $\tau$. Let $h_{\mathcal{A}, \mathcal{A}^{\prime}}$ : $\mathcal{A}^{\prime} \rightarrow \mathbb{R}$ be any lifting function which is zero on $\mathcal{A}$ and positive otherwise. Extending $h_{\tau}$ by zero outside $\mathcal{A}$, we get that for $\epsilon>0$ small enough the function $h^{\prime}:=h_{\mathcal{A}, \mathcal{A}^{\prime}}+\epsilon \cdot h_{\tau}$ induces a regular subdivision $\tau^{\prime}$ of $\mathcal{A}^{\prime}$ containing the cells in $\tau$ and $h:=\left.h^{\prime}\right|_{\mathcal{A}}=\epsilon \cdot h_{\tau}$ induces $\tau$.

Lemma A.2. Let $\mathcal{A} \subset \mathcal{A}^{\prime} \subset \mathbb{Z}^{d^{\prime}}$ be finite point configurations, with $\operatorname{rkAff}(\mathcal{A})=d<$ $\operatorname{rkAff}\left(\mathcal{A}^{\prime}\right)=d^{\prime}$. Assume moreover that $\mathcal{A}^{\prime} \backslash \mathcal{A}$ has cardinality $d^{\prime}-d$. Suppose that $\tau$ is a regular subdivision (triangulation) of $\mathcal{A}$. For each $\sigma \in \tau$ define $\sigma^{\prime}=\sigma \cup\left(\mathcal{A}^{\prime} \backslash \mathcal{A}\right)$. Then, the collection $\tau^{\prime}:=\left\{\sigma^{\prime}, \sigma \in \tau\right\}$ defines a regular subdivision (triangulation) of $\mathcal{A}^{\prime}$. Moreover, $\tau^{\prime}$ can be induced by a lifting function $h^{\prime}$ whose restriction to $\mathcal{A}$ induces $\tau$.

Proof. Consider a point $a \in \mathcal{A}^{\prime} \backslash \mathcal{A}$. Then $a$ is outside the hyperplane spanned by $\mathcal{A}$, that is, $a \cup \mathcal{A}$ is a pyramid over $\mathcal{A}$. It is known (see Observation 4.2.3 in [12]) that the collection $\{\sigma \cup a, \sigma \in \tau\}$ is a subdivision of $a \cup \mathcal{A}$, and it is regular if and only if $\tau$ is regular. Then, we can see $\mathcal{A}^{\prime}$ as an iterated pyramid over $\mathcal{A}$ and the lemma follows by applying successively the previous fact.

Given a matrix $D \in \mathbb{R}^{d_{D} \times n_{D}}$ and $I \subset\left\{1, \ldots, n_{D}\right\}$, we will denote by $D_{I}$ the submatrix of $D$ corresponding to the columns indexed by $I$. For $i \in\left\{1, \ldots, n_{D}\right\}, D(i)$ will denote the matrix obtained by removing the $i$-th column of $D$, and for $j \in\left\{1, \ldots, d_{D}\right\}, D_{j}$ will denote the $j$-th row of $D$.

Theorem A.3. Let $d, d^{\prime} \in \mathbb{N}$ with $d \leq d^{\prime}$. Let $\mathcal{A} \subset \mathcal{A}^{\prime \prime} \subset \mathbb{Z}^{d^{\prime}}$ be finite point configurations, with $\operatorname{rkAff}(\mathcal{A})=d, \operatorname{rkAff}\left(\mathcal{A}^{\prime \prime}\right)=d^{\prime}$. Write $\mathcal{A}=\left\{a_{1}, \ldots, a_{n}\right\}, \mathcal{A}^{\prime \prime}=\mathcal{A} \cup\left\{a_{n+1}, \ldots, a_{m}\right\}$, with $m \geq d^{\prime}>n$. Set $\mathcal{A}^{\prime}=\mathcal{A} \cup\left\{a_{n+1}, \ldots, a_{n+d^{\prime}-d}\right\}$ and assume that rkAff $\mathcal{A}^{\prime}=d^{\prime}$. Let $\tau$ be a regular subdivision of $\mathcal{A}$ induced by a lifting function $h, \tau^{\prime}$ the regular subdivision of $\mathcal{A}^{\prime}$ obtained as in Lemma A.2, and $\tau^{\prime \prime}$ any regular subdivision of $\mathcal{A}^{\prime \prime}$ such that $\tau^{\prime} \subset \tau^{\prime \prime}$, induced by a lifting function $h^{\prime \prime}$, such that $h^{\prime \prime}$ restricted to $\mathcal{A}$ induces $\tau$.

Let $f_{1}, \ldots, f_{d}$ be polynomials with support in $\mathcal{A}$ and coefficient matrix $C$ of rank $d$. Let $f_{1}^{\prime \prime}, \ldots, f_{d}^{\prime \prime}, \ldots, f_{d^{\prime}}^{\prime \prime}$ be polynomials with support $\mathcal{A}^{\prime \prime}$ and coefficient matrix $C^{\prime \prime}$ of rank $d^{\prime}$ of the form

$$
\left(\begin{array}{lll}
C & 0 & D_{1} \\
M & B & D_{2}
\end{array}\right)
$$

with $B \in \mathbb{R}^{\left(d^{\prime}-d\right) \times\left(d^{\prime}-d\right)}$ invertible. Assume $\tau$ has $p$ d-simplices positively decorated by $C$ and the determinants of the submatrices

$$
\left(\begin{array}{c}
C_{I} \\
\left(B^{-1} M_{I}\right)_{j}
\end{array}\right)
$$

have all the same sign as $(-1)^{d+i} \operatorname{det}\left(C_{I}(i)\right)$, for each $i=1, \ldots, d+1$, for each $j=$ $1, \ldots, d^{\prime}-d$, and for each subset $I \subset\{1, \ldots, n\}$ which indexes a positively decorated simplex. Then, there exists $t_{0}>0$, such that for $0<t<t_{0}$, the deformed system $f_{1, t}^{\prime \prime}=\cdots=f_{d^{\prime}, t}^{\prime \prime}=0$, where

$$
f_{i, t}^{\prime \prime}(x)=\sum_{j=1}^{m} c_{i, j}^{\prime \prime} t^{h^{\prime \prime}\left(a_{j}\right)} x^{a_{j}},
$$

has at least $p$ positive nondegenerate real roots. 
Proof. The subdivision $\tau^{\prime \prime}$ can be obtained by Lemma A.1. Note that the columns of $B$ correspond to the points in $\mathcal{A}^{\prime} \backslash \mathcal{A}$.

Suppose that $\Delta \in \tau$ is a $d$-simplex positively decorated by $C$. Then $\Delta^{\prime}=\Delta \cup$ $\left\{a_{n+1}, \ldots, a_{n+d^{\prime}-d}\right\}$ is a $d^{\prime}$-simplex of $\tau^{\prime} \subset \tau^{\prime \prime}$. We will show that $\Delta^{\prime}$ is positively decorated by $C^{\prime \prime}$.

Suppose that $\Delta$ is indexed by the set $I=\left\{i_{1}, \ldots, i_{d+1}\right\}$ of $\{1, \ldots, n\}$. We have to prove that the submatrix $C_{I^{\prime}}^{\prime \prime}$ of $C^{\prime \prime}$ is positively spanning, with $I^{\prime}=I \cup\left\{n+1, \ldots, n+d^{\prime}-d\right\}$. This is equivalent to prove that the matrix

$$
G=\left(\begin{array}{cc}
\operatorname{Id}_{\mathrm{d}} & 0 \\
0 & B^{-1}
\end{array}\right)\left(\begin{array}{cc}
C_{I} & 0 \\
M_{I} & B
\end{array}\right)=\left(\begin{array}{cc}
C_{I} & 0 \\
B^{-1} M_{I} & \operatorname{Id}_{d^{\prime}-d}
\end{array}\right),
$$

is positively spanning, as the property of being positively spanning remains invariant under multiplication by invertible matrices.

We compute $(-1)^{i} \operatorname{det}(G(i))$ for $i=1, \ldots, d^{\prime}+1$. For $i=1, \ldots, d+1$, we have:

$$
(-1)^{i} \operatorname{det}(G(i))=(-1)^{i} \operatorname{det}\left(\begin{array}{cc}
C_{I}(i) & 0 \\
B^{-1} M_{I}(i) & \operatorname{Id}_{\mathrm{d}^{\prime}-\mathrm{d}}
\end{array}\right)=(-1)^{i} C_{I}(i),
$$

which have all the same sign, because $\Delta$ is positively decorated by $C$. Take now $i>d+1$. Let $j \in 1, \ldots, d^{\prime}-d$ such that $d+1+j=i$. Moving the $i$-th row of $G$ to the row $d+2$ in $j-1$ interchanges of consecutive rows, we have:

$$
\begin{aligned}
(-1)^{i} \operatorname{det}(G(i)) & =(-1)^{i} \operatorname{det}\left(\begin{array}{cc}
C_{I} & 0 \\
B^{-1} M_{I} & \operatorname{Id}_{\mathrm{d}^{\prime}-\mathrm{d}}(\mathrm{j})
\end{array}\right) \\
& =(-1)^{d+1+j}(-1)^{j-1} \operatorname{det}\left(\begin{array}{cc}
C_{I} & 0 \\
\left(B^{-1} M_{I}\right)_{j} & 0 \\
\left(B^{-1} M_{I}\right)[j] & \operatorname{Id}_{\mathrm{d}^{\prime}-\mathrm{d}-1}(\mathrm{j})
\end{array}\right) \\
& =(-1)^{d} \operatorname{det}\left(\begin{array}{c}
C_{I} \\
\left(B^{-1} M_{I}\right)_{j}
\end{array}\right),
\end{aligned}
$$

where $\left(B^{-1} M_{I}\right)[j]$ denotes the submatrix of $B^{-1} M_{I}$ obtained by removing its $j$-th row. For each $j=1, \ldots, d^{\prime}-d$, this determinant has the same sign as $(-1)^{i} C_{I}(i)$, for each $j=1, \ldots, d^{\prime}-d$, by hypothesis. Then, the simplex $\Delta^{\prime}$ is positively decorated by $C^{\prime \prime}$.

We deduce that if $\tau$ has $p d$-simplices positively decorated by $C$, then $\tau^{\prime \prime}$ has $p d^{\prime}$ simplices positively decorated by $C^{\prime \prime}$. Then, by Theorem 2.9 in citeAGB1 (from which we extracted 2.7), there exists $t_{0}>0$, such that for $0<t<t_{0}$, the system $f_{1, t}^{\prime \prime}=\cdots=f_{d^{\prime}, t}^{\prime \prime}=$ 0 , has at least $p$ positive nondegenerate real roots.

Remark A.4. The conditions that guarantee that the $p d^{\prime}$-simplices of $\tau^{\prime \prime}$ are positively decorated by $C^{\prime \prime}$ include the conditions such that the $p d$-simplices of $\tau$ are positively decorated by $C$, plus other conditions. In the cases of cascades of Goldbeter-Koshland loops that we studied in Section 4, these other conditions are automatically fulfilled.

Dto. de Matemática, FCen, Universidad de Buenos Aires, and imas (UBA-COniCET), Ciudad Universitaria, Pab. I, C1428Ega Buenos Aires, Argentina

E-mail address: mgiaroli@dm.uba.ar

Laboratoire de Mathématiques, Université Savoie Mont Blanc, 73376 Le Bourget-du-LaC Cedex, France

E-mail address: Frederic.Bihan@univ-savoie.fr

URL: http://www. lama.univ-savoie.fr/ bihan/

Dto. de Matemática, FCEn, Universidad de Buenos Aires, and imas (UBA-CONiCET), Ciudad Universitaria, Pab. I, C1428EGA Buenos Aires, Argentina

E-mail address: alidick@dm.uba.ar

URL: http://mate.dm.uba.ar/〜alidick 